\title{
AN EDDY CURRENT PROBLEM IN TERMS OF A TIME-PRIMITIVE OF THE ELECTRIC FIELD WITH NON-LOCAL SOURCE CONDITIONS*
}

\author{
Alfredo Bermúdez ${ }^{1}$, Bibiana López-Rodríguez ${ }^{2}$, Rodolfo Rodríguez ${ }^{3}$ \\ AND PILAR SALGADO ${ }^{4}$
}

\begin{abstract}
The aim of this paper is to analyze a formulation of the eddy current problem in terms of a time-primitive of the electric field in a bounded domain with input current intensities or voltage drops as source data. To this end, we introduce a Lagrange multiplier to impose the divergence-free condition in the dielectric domain. Thus, we obtain a time-dependent weak mixed formulation leading to a degenerate parabolic problem which we prove is well-posed. We propose a finite element method for space discretization based on Nédélec edge elements for the main variable and standard finite elements for the Lagrange multiplier, for which we obtain error estimates. Then, we introduce a backward Euler scheme for time discretization and prove error estimates for the fully discrete problem, too. Finally, the method is applied to solve a couple of test problems.
\end{abstract}

Mathematics Subject Classification. 65N30, 78A25.

Received June 15, 2012. Revised November 6, 2012.

Published online April 17, 2013.

\section{INTRODUCTION}

The goal of this paper is to analyze a time-dependent eddy current problem defined in a three-dimensional bounded domain including conducting and dielectric materials, subject to source boundary conditions feasible from the physical point of view. This model arises in applications where the problem is reduced to a bounded domain and it is necessary to link the electromagnetic fields with sources provided by an external circuit by

Keywords and phrases. Eddy current problems, time-dependent electromagnetic problems, input current intensities, voltage drops, finite elements..

* The work of the authors from Universidade de Santiago de Compostela was supported by FEDER/MTM2008-02483, Ministerio de Ciencia e Innovación (Spain), and INCITE09-207047-PR and Grupos de Referencia Competitiva, Xunta de Galicia (Spain). R. Rodríguez was partially supported by BASAL project CMM, Universidad de Chile (Chile). B. López-Rodríguez was supported by MECESUP UCO0713 (Chile), Banco Santander-USC fellowship (Spain), and projects 201010010107 and 20101009545, Vicerrectoría de Investigación, Universidad Nacional de Colombia (Colombia).

1 Departamento de Matemática Aplicada, Universidad de Santiago de Compostela, 15706, Santiago de Compostela, Spain. alfredo.bermudez@usc.es

2 Escuela de Matemáticas, Universidad Nacional de Colombia, sede Medellín, Colombia. blopezr@unal.edu.co

$3 \mathrm{CI}^{2} \mathrm{MA}$, Departamento de Ingeniería Matemática, Universidad de Concepción, Casilla 160-C, Concepción, Chile. rodolfo@ing-mat.udec.cl

4 Departamento de Matemática Aplicada, Escola Politécnica Superior, Universidade de Santiago de Compostela, 27002, Lugo, Spain. mpilar.salgado@usc.es 
means of current intensities or voltage drops (see, for instance, $[13,20]$ ). Both cases of source data will be separately analyzed in domains with a rather complex geometry, which allows modeling a great variety of applications.

In the literature, we can find some papers related to the numerical analysis of the three-dimensional time-dependent eddy current model in bounded domains containing conducting and dielectric materials $[1,9,15,23,24,28]$. Most of these articles deal with the case where the conducting materials are strictly contained in the computational domain and the source current is imposed in an inner subdomain. These formulations involve natural and/or essential boundary conditions which differ depending on the primary unknown. However, to the best of the authors knowledge, the reference for the mathematical analysis of transient problems with current or voltage excitations is [9].

In this reference, the current sources can be intensities or voltages, which are imposed by means of non-local boundary conditions (see [6] for the corresponding problem in harmonic regime). The problem is written in terms of the magnetic field, which must satisfy the curl-free condition in the dielectric domain. At the discrete level, a magnetic scalar potential is introduced in the dielectric domain, which allows an important saving in computational effort. However, this formulation requires to build "cutting" surfaces to make the dielectric domain simply connected. These cutting surfaces can be difficult to build in complex geometries. In harmonic regime, several alternatives have been proposed to avoid the construction of these surfaces when the current sources are imposed in an inner subdomain [2,3] and when the sources are provided by external circuits [8].

The present paper analyzes a formulation of the problem based on the time-primitive of the electric field and a Lagrange multiplier to impose the divergence-free constraint of the electric displacement. Although the computer cost is significantly more expensive than that of the method proposed in [9], this new contribution has the relevant feature that it can be applied to more complex geometries, since the scalar magnetic potential is not introduced in the dielectric domain and therefore cutting surfaces are not needed.

The time-primitive of the electric field has been introduced in the literature of electrical engineering in [16] and it is usually known as modified magnetic vector potential. This potential has been used by other authors (see, for instance, $[21,22,26]$ ) who usually couple this vector field with different unknowns in the dielectric part, leading to degenerate parabolic problems. The use of this variable in eddy current problems with inner current sources has been analyzed in [1] (see also [15]). However, replacing internal current density sources with current/voltage excitations, which are of non-local nature, makes the problem more difficult. In fact, the authors were not able to prove the well-posedness of the continuous and discrete problems by adapting the arguments in [1]. This is why an alternative and indirect proof has been written based on the formulation in magnetic field (see [9]) and on previous results for magnetostatiscs problems from [17].

Thus, in order to analyze the resulting weak formulation, we resort to some results obtained in [9] for the magnetic field formulation of the same problem. In particular we use that the weak solution obtained in this reference satisfies the eddy current model initially posed. This fact allows us to show that the time-primitive of the corresponding electric field on the conducting domain leads to a solution of the present formulation.

As in [1], the formulation analyzed in this paper needs as a data the normal component of the electric displacement on the outer boundary. However, we prove an interesting fact: this boundary data has no effect on the value of the main physical quantities, namely, the magnetic field in the whole domain and the electric field in the conducting one. Thus, the data actually needed in practice reduces to input current intensities or voltage drops, which are the genuine data of our problem.

To discretize the mixed problem we propose a finite element method on tetrahedral meshes based on Nédélec edge elements for the main variable and standard piecewise linear elements for the Lagrange multiplier. We show that this leads to a degenerate algebraic-differential system. To prove that this problem is well-posed, we need to build a non standard basis for the discrete kernel which allows us to conclude that the algebraicdifferential system has a unique solution. Although in principle this solution has a square integrable timederivative only in the conducting domain, we actually prove that this holds on the dielectric too. This fact allows us to obtain optimal order error estimates. Finally we introduce an implicit time discretization. We resort 
to similar arguments as in the semidiscrete case for proving that the problem is well posed. Then, we obtain optimal order error estimates in this case, too.

Let us remark that under the assumption of time-independent electromagnetic coefficients, similar arguments lead to a formulation in terms of the electric field, too. In principle the techniques in this paper look promising to analyze such a formulation, provided further smoothness in time holds for the boundary data.

The outline of the paper is as follows. In Section 2 we introduce the transient eddy current model and state the geometrical framework for the analysis. In Section 3 we analyze the problem with input current intensities as boundary data. We obtain a time-dependent weak mixed formulation of the problem with input current intensities as boundary data and prove that it is well-posed. We introduce a space discretization based on finite elements and prove error estimates. We propose a backward Euler scheme for time discretization and obtain error estimates for the fully discretized problem. In Section 4 we perform a similar analysis for the transient eddy current problem, but now with voltage drops as boundary data. In Section 5, we report some numerical results. We present a test with known analytical solution which allows us to confirm the order of convergence predicted by the theory in both cases, namely, using intensities or voltage drops as source data. Finally, we apply the method to an application which involves a more complex geometry.

\section{Statement of the PROBlem}

Three dimensional eddy current problems describe low-frequency electromagnetic phenomena. In this case, displacement currents may be neglected (see, for instance, [12], Chap. 8), so that Maxwell's equations restricted to a domain $\Omega$ become

$$
\begin{aligned}
\operatorname{curl} \boldsymbol{H}=\boldsymbol{J} & \text { in }[0, T] \times \Omega, \\
\partial_{t}(\mu \boldsymbol{H})+\operatorname{curl} \boldsymbol{E}=\mathbf{0} & \text { in }[0, T] \times \Omega, \\
\operatorname{div}(\mu \boldsymbol{H})=0 & \text { in }[0, T] \times \Omega, \\
\boldsymbol{J}=\sigma \boldsymbol{E} & \text { in }[0, T] \times \Omega,
\end{aligned}
$$

where $\boldsymbol{E}(t, \boldsymbol{x})$ is the electric field, $\boldsymbol{H}(t, \boldsymbol{x})$ the magnetic field, $\boldsymbol{J}(t, \boldsymbol{x})$ the current density, $\mu$ the magnetic permeability and $\sigma$ the electric conductivity. Here and thereafter, we use boldface letters to denote vector fields and variables as well as vector-valued operators.

We assume that $\Omega$ is a simply connected three-dimensional bounded domain, which consists of two parts, $\Omega_{\mathrm{C}}$ and $\Omega_{\mathrm{D}}$, occupied by conductors and dielectrics, respectively. We assume that $\Omega_{\mathrm{D}}$ is connected. The domain $\Omega$ is assumed to have a Lipschitz-continuous connected boundary. We denote by $\Gamma_{\mathrm{C}}, \Gamma_{\mathrm{D}}$ and $\Gamma_{\mathrm{I}}$ the open surfaces such that $\bar{\Gamma}_{\mathrm{C}}:=\partial \Omega_{\mathrm{C}} \cap \partial \Omega$ is the outer boundary of the conductor domain, $\bar{\Gamma}_{\mathrm{D}}:=\partial \Omega_{\mathrm{D}} \cap \partial \Omega$ that of the dielectric domain and $\bar{\Gamma}_{\mathrm{I}}:=\partial \Omega_{\mathrm{C}} \cap \partial \Omega_{\mathrm{D}}$ the interface between both domains. We also denote by $\boldsymbol{n}, \boldsymbol{n}_{\mathrm{C}}$ and $\boldsymbol{n}_{\mathrm{D}}$ the outer unit normal vectors to $\partial \Omega, \partial \Omega_{\mathrm{C}}$ and $\partial \Omega_{\mathrm{D}}$, respectively. Notice that $\boldsymbol{n}_{\mathrm{C}}=\boldsymbol{n}$ on $\Gamma_{\mathrm{C}}, \boldsymbol{n}_{\mathrm{D}}=\boldsymbol{n}$ on $\Gamma_{\mathrm{D}}$ and $\boldsymbol{n}_{\mathrm{C}}=-\boldsymbol{n}_{\mathrm{D}}$ on $\Gamma_{\mathrm{\Gamma}}$.

As shown in Figure 1, the disjoint connected components of the conducting domain are of two types: "inductors" which go through the boundary of $\Omega$, and "workpieces" which have their closure included in $\Omega$. We denote $\Omega_{\mathrm{C}}^{1}, \ldots, \Omega_{\mathrm{C}}^{N}$ the former and $\Omega_{\mathrm{C}}^{N+1}, \ldots, \Omega_{\mathrm{C}}^{M}$ the latter. Moreover, we assume that each $\Omega_{\mathrm{C}}^{n}, n=1, \ldots, M$, is simply connected with a connected boundary $\partial \Omega_{\mathrm{C}}^{n}$. We assume that $\Gamma_{\mathrm{I}}$ splits in connected components as follows: $\Gamma_{\mathrm{I}}=\bigcup_{n=1}^{M} \Gamma_{\mathrm{I}}^{n}$, where $\Gamma_{\mathrm{I}}^{n}:=\Gamma_{\mathrm{I}} \cap \partial \Omega_{\mathrm{C}}^{n}, n=1, \ldots, M$.

We assume that the outer boundary of each inductor, $\partial \Omega_{\mathrm{C}}^{n} \cap \partial \Omega, n=1, \ldots, N$, has two disjoint connected components, both being the closure of open simply connected surfaces: the current entrance $\Gamma_{\mathrm{J}}^{n}$, where the inductor is connected to a transient electric current source, and the current exit $\Gamma_{\mathrm{E}}^{n}$. We denote $\Gamma_{\mathrm{J}}:=\Gamma_{\mathrm{J}}^{1} \cup \cdots \cup \Gamma_{\mathrm{J}}^{N}$ and $\Gamma_{\mathrm{E}}:=\Gamma_{\mathrm{E}}^{1} \cup \cdots \cup \Gamma_{\mathrm{E}}^{N}$. Furthermore, we assume that $\bar{\Gamma}_{\mathrm{J}}^{n} \cap \bar{\Gamma}_{\mathrm{J}}^{m}=\emptyset, \bar{\Gamma}_{\mathrm{E}}^{n} \cap \bar{\Gamma}_{\mathrm{E}}^{m}=\emptyset, 1 \leq m, n \leq N, m \neq n$, and $\bar{\Gamma}_{\mathrm{J}} \cap \bar{\Gamma}_{\mathrm{E}}=\emptyset$.

We assume that for each inductor, $\Omega_{\mathrm{C}}^{n}, n=1, \ldots, N$, there exists one connected "cut" surface $\Sigma_{n} \subset \Omega_{\mathrm{D}}$, with $\partial \Sigma_{n} \subset \partial \Omega_{\mathrm{C}}^{n} \cup \Gamma_{\mathrm{D}}$, such that $\widetilde{\Omega}_{\mathrm{D}}:=\Omega_{\mathrm{D}} \backslash \bigcup_{n=1}^{N} \Sigma_{n}$ is pseudo-Lipschitz and simply connected (see, for instance, [7]). 


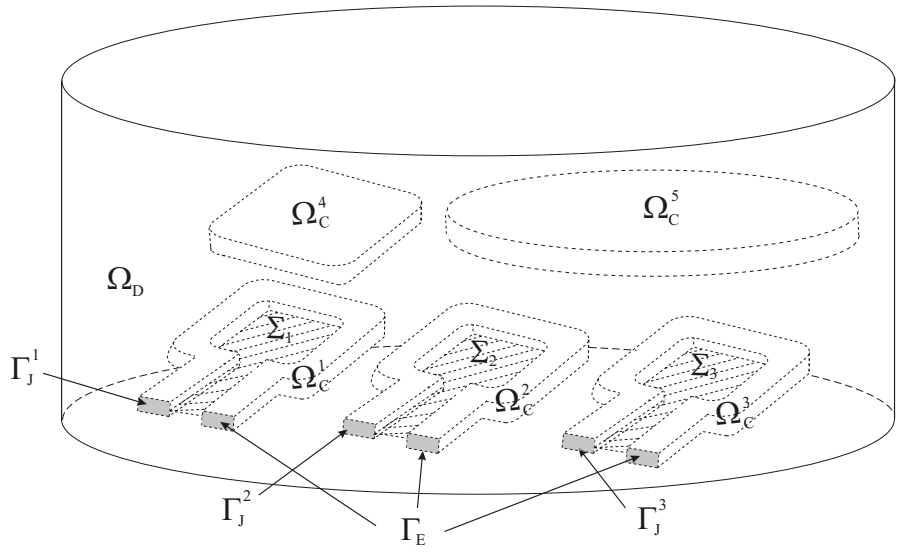

FIGURE 1. Sketch of the domain.

We also assume that $\bar{\Sigma}_{n} \cap \bar{\Sigma}_{m}=\emptyset$ for $n \neq m$ (see Fig. 1). We denote $\Sigma:=\bigcup_{n=1}^{N} \Sigma_{n}$ and assume that $\Gamma_{\mathrm{D}}$ and $\Gamma_{\mathrm{D}} \backslash \partial \Sigma$ are connected.

We suppose that $\mu$ and $\sigma$ are time-independent and there exist positive constants $\underline{\mu}, \bar{\mu}, \bar{\sigma}$ and $\underline{\sigma}$ such that

$$
\begin{aligned}
& 0<\underline{\mu} \leq \mu(\boldsymbol{x}) \leq \bar{\mu}, \quad \text { a.e. } \boldsymbol{x} \in \Omega, \\
& 0<\underline{\sigma} \leq \sigma(\boldsymbol{x}) \leq \bar{\sigma}, \quad \text { a.e. } \boldsymbol{x} \in \Omega_{\mathrm{C}} \quad \text { and } \quad \sigma \equiv 0 \text { in } \Omega_{\mathrm{D}} .
\end{aligned}
$$

We have to complete the model with an initial condition, $\boldsymbol{H}(0)=\boldsymbol{H}_{0}$, the source terms and suitable boundary conditions. For the latter, we consider the following ones:

$$
\begin{array}{cl}
\boldsymbol{E} \times \boldsymbol{n}=\mathbf{0} & \text { on }[0, T] \times \Gamma_{\mathrm{E}}, \\
\boldsymbol{E} \times \boldsymbol{n}=\mathbf{0} & \text { on }[0, T] \times \Gamma_{\mathrm{J}}, \\
\mu \boldsymbol{H} \cdot \boldsymbol{n}=0 & \text { on }[0, T] \times \partial \Omega .
\end{array}
$$

Conditions (2.5), (2.6) and (2.7) have been proposed in [13] in a more general setting. They will appear as natural boundary conditions of our weak formulation of the problem. The former mean that the electric current density is normal to the current entrance and exit surfaces, whereas the latter means that the magnetic field is tangential to the boundary of the whole domain $\Omega$.

To consider sources provided by external circuits we have two possibilities: either the intensities of the input current or the voltage drops most be given for each inductor $\Omega_{\mathrm{C}}^{n}, n=1, \ldots, N$. In the first case, from (2.4), we have that

$$
\int_{\Gamma_{\mathrm{J}}^{n}} \sigma \boldsymbol{E} \cdot \boldsymbol{n}=I_{n} \quad \text { in }[0, T]
$$

where $I_{n}$ is the current intensity through the surface $\Gamma_{\mathrm{J}}^{n}$.

To write down the equation corresponding to the second case, let $V_{n}$ be the input voltage drop along the inductor $\Omega_{\mathrm{C}}^{n}$. It follows from (2.7) and (2.2) that $\operatorname{curl} \boldsymbol{E} \cdot \boldsymbol{n}=0$ on $[0, T] \times \partial \Omega$. Hence, there exists a surface potential $V(t, \boldsymbol{x})$ defined on the boundary of the whole $\Omega$ and such that $\boldsymbol{n} \times \boldsymbol{E}(t, \boldsymbol{x}) \times \boldsymbol{n}=-\operatorname{grad}_{\tau} V(t, \boldsymbol{x})$ on $\partial \Omega$, where $\operatorname{grad}_{\tau}$ denotes the surface gradient ( $c f$. [14]). Moreover, (2.5) and (2.6) imply that $V(t, \boldsymbol{x})$ must be constant on each connected component of $\Gamma_{\mathrm{J}}$ and $\Gamma_{\mathrm{E}}$. The difference $V_{n}(t):=\left.V\right|_{\Gamma_{\mathrm{E}}^{n}}(t)-\left.V\right|_{\Gamma_{\mathrm{J}}^{n}}(t)$ is the voltage drop along the conductor $\Omega_{\mathrm{C}}^{n}$. Thus, given $V_{n}$, the boundary condition reads

$$
\boldsymbol{n} \times \boldsymbol{E} \times \boldsymbol{n}=-\operatorname{grad}_{\tau} V \text { on } \partial \Omega, \text { with }\left.V\right|_{\Gamma_{\mathrm{E}}^{n}}-\left.V\right|_{\Gamma_{\mathrm{J}}^{n}}=V_{n} \quad \text { in }[0, T] .
$$


Although in a same problem we could consider that voltage drops are known for some inductors and current intensities for the others, for simplicity we will study each case separately.

We have shown in [9] that equations (2.1)-(2.7) with boundary data (2.8) or (2.9) and initial condition $\boldsymbol{H}_{0}$ satisfying appropriate assumptions, lead to a well-posed problem. Note that, since the electric conductivity coefficient $\sigma$ vanishes in $\Omega_{\mathrm{D}}$, we do not have uniqueness of the electric field $\boldsymbol{E}$ in $\Omega_{\mathrm{D}}$; in fact, if we add to a solution $\boldsymbol{E}$ the gradient of any function with compact support in $\Omega_{\mathrm{D}}$, the resulting field also solves (2.1)-(2.7).

Therefore, we must add equations so that $\boldsymbol{E}$ is uniquely determined. With this aim we introduce the following conditions as in $[1,5]$ which assumes absence of electric charge in the dielectric domain:

$$
\begin{aligned}
\operatorname{div}(\epsilon \boldsymbol{E})=0 \quad \text { in } \quad[0, T] \times \Omega_{\mathrm{D}}, \\
\left.\epsilon \boldsymbol{E}\right|_{\Omega_{\mathrm{D}}} \cdot \boldsymbol{n}=g \quad \text { on }[0, T] \times \Gamma_{\mathrm{D}}, \\
\left.\int_{\Gamma_{\mathrm{I}}^{k}} \epsilon \boldsymbol{E}\right|_{\Omega_{\mathrm{D}}} \cdot \boldsymbol{n}=0, \quad k=2, \ldots, M, \quad \text { in }[0, T],
\end{aligned}
$$

where $\epsilon$ is the electric permittivity and $g$ is an additional data. Notice that $\left.\int_{\Gamma_{\mathrm{I}}^{1}} \epsilon \boldsymbol{E}\right|_{\Omega_{\mathrm{D}}} \cdot \boldsymbol{n}$ is also fixed. In fact, from the equations above and Gauss Theorem, $\left.\int_{\Gamma_{\mathrm{I}}^{1}} \epsilon \boldsymbol{E}\right|_{\Omega_{\mathrm{D}}} \cdot \boldsymbol{n}=-\int_{\Gamma_{\mathrm{D}}} g$.

Boundary condition (2.11) involves the knowledge of an additional boundary data, the normal trace of $\epsilon \boldsymbol{E}$ on $\Gamma_{\mathrm{D}}$, which can be difficult to obtain in practice. However, we prove in the present paper that $\left.\boldsymbol{E}\right|_{\Omega_{\mathrm{C}}}$ and $\boldsymbol{H}$ in the whole domain $\Omega$ are independent of the value of $g$. Hence, this allows us to choose, for instance, $g=0$ in (2.11) if we are not interested in the electric field in $\Omega_{\mathrm{D}}$ (see Rem. 3.5). In such a case, $\left.\boldsymbol{E}\right|_{\Omega_{\mathrm{D}}}$ is not the actual electric field but just an auxiliary variable which allows us to compute the typical quantities of interest: $\left.\boldsymbol{E}\right|_{\Omega_{\mathrm{C}}}$ and $\boldsymbol{H}$.

Throughout this paper, we will use standard notation for Sobolev spaces and norms. We will also use the well known Hilbert spaces $\mathrm{H}(\operatorname{curl} ; \Omega), \mathrm{H}(\operatorname{div} ; \Omega), \mathrm{H}_{0}\left(\operatorname{div}^{0} ; \Omega\right)$, etc. (see, for instance, [7]).

Let us remark that, given $\eta$ and $\varsigma \in \mathrm{H}^{-1 / 2}\left(\partial \Omega_{\mathrm{D}}\right)$, we say that $\eta=\varsigma$ on $\Gamma$, where $\Gamma$ is an open surface contained in $\partial \Omega_{\mathrm{D}}$, if $\eta=\varsigma$ on $\mathrm{H}_{00}^{-1 / 2}(\Gamma)$; namely, if $\langle\eta, \phi\rangle_{\partial \Omega_{\mathrm{D}}}=\langle\varsigma, \phi\rangle_{\partial \Omega_{\mathrm{D}}} \forall \phi \in \mathrm{H}_{00}^{1 / 2}(\Gamma)$, where $\langle\cdot, \cdot\rangle_{\partial \Omega_{\mathrm{D}}}$ denotes the duality pairing in $\mathrm{H}^{-1 / 2}\left(\partial \Omega_{\mathrm{D}}\right) \times \mathrm{H}^{1 / 2}\left(\partial \Omega_{\mathrm{D}}\right)$. In particular, equation $(2.11)$ must be understood in this sense. Similarly, equation (2.8) has to be understood as the a duality paring $\langle\sigma \boldsymbol{E}(t) \cdot \boldsymbol{n}, 1\rangle_{\Gamma_{\mathrm{J}}^{n}}$. This paring is well defined because $\sigma \boldsymbol{E}(t) \cdot \boldsymbol{n}=0$ on $\Gamma_{\mathrm{D}}$ (see, [17] Prop. 3.3). The same happens with equation (2.12).

\section{EDDY CURRENT PROBLEM WITH INPUT CURRENT INTENSITIES AS SOURCE DATA}

The aim of this section is to analyze a formulation in terms of a time-primitive of the electric field of the transient eddy current problem given by equations $(2.1)-(2.8)$, the latter for $n=1, \ldots, N$, and $(2.10)-(2.12)$ with an adequate initial condition $\boldsymbol{H}_{0}$, under appropriate assumptions of the data. In particular, we assume that $g \in \mathrm{L}^{2}\left(0, T ; \mathrm{L}^{2}\left(\Gamma_{\mathrm{D}}\right)\right), I_{n} \in \mathrm{H}^{2}(0, T), n=1, \ldots, N$, and the initial data $\boldsymbol{H}_{0}$ satisfies

$$
\boldsymbol{H}_{0} \in \mathcal{X}, \quad\left\langle\operatorname{curl} \boldsymbol{H}_{0} \cdot \boldsymbol{n}, 1\right\rangle_{\Gamma_{\mathrm{J}}^{n}}=I_{n}(0), n=1, \ldots, N, \quad \text { and } \quad \mu \boldsymbol{H}_{0} \in \mathrm{H}_{0}\left(\operatorname{div}^{0} ; \Omega\right),
$$

where

$$
\mathcal{X}:=\left\{\boldsymbol{G} \in \mathrm{H}(\operatorname{curl} ; \Omega): \operatorname{curl} \boldsymbol{G}=\mathbf{0} \text { in } \Omega_{\mathrm{D}}\right\} .
$$

Let us introduce the time-primitive of the electric field

$$
\boldsymbol{u}(t, \boldsymbol{x}):=\int_{0}^{t} \boldsymbol{E}(s, \boldsymbol{x}) \mathrm{d} s .
$$

Integrating $(2.2)$ over $[0, t]$ we obtain

$$
\mu(\boldsymbol{x}) \boldsymbol{H}(t, \boldsymbol{x})-\mu(\boldsymbol{x}) \boldsymbol{H}_{0}(\boldsymbol{x})+\operatorname{curl} \boldsymbol{u}(t, \boldsymbol{x})=\mathbf{0} .
$$


Using that $\boldsymbol{u}(0, \boldsymbol{x})=\mathbf{0}$, it is easy to write the transient eddy current equations in terms of $\boldsymbol{u}$ as follows:

$$
\begin{aligned}
\sigma \partial_{t} \boldsymbol{u}+\operatorname{curl}\left(\frac{1}{\mu} \operatorname{curl} \boldsymbol{u}\right)=\operatorname{curl} \boldsymbol{H}_{0} & \text { in }[0, T] \times \Omega, \\
\operatorname{curl} \boldsymbol{u} \cdot \boldsymbol{n}=0 & \text { on }[0, T] \times \partial \Omega, \\
\boldsymbol{u} \times \boldsymbol{n}=\mathbf{0} & \text { on }[0, T] \times \Gamma_{\mathrm{C}}, \\
\operatorname{div}(\epsilon \boldsymbol{u})=0 & \text { in }[0, T] \times \Omega_{\mathrm{D}}, \\
\epsilon \boldsymbol{u}(t) \cdot \boldsymbol{n}=\int_{0}^{t} g(s) \text { d } s & \text { on } \Gamma_{\mathrm{D}}, \quad t \in[0, T], \\
\left\langle\left.\epsilon \boldsymbol{u}\right|_{\Omega_{\mathrm{D}}} \cdot \boldsymbol{n}, 1\right\rangle_{\Gamma_{\mathrm{I}}^{k}}=0, & k=2, \ldots, M, \quad \text { in }[0, T], \\
\langle\sigma \boldsymbol{u}(t) \cdot \boldsymbol{n}, 1\rangle_{\Gamma_{\mathrm{J}}^{n}}=\int_{0}^{t} I_{n}(s) \mathrm{d} s, & n=1, \ldots, N, \quad t \in[0, T], \\
\boldsymbol{u}(0)=\mathbf{0} & \text { in } \Omega .
\end{aligned}
$$

Our next goal is to obtain a weak formulation of this problem. With this end, we introduce the following space:

$$
\mathcal{U}:=\left\{\boldsymbol{w} \in \mathrm{H}(\operatorname{curl} ; \Omega): \boldsymbol{w} \times \boldsymbol{n}=\mathbf{0} \text { on } \Gamma_{\mathrm{C}} \text { and } \operatorname{curl} \boldsymbol{w} \cdot \boldsymbol{n}=0 \text { on } \partial \Omega\right\} .
$$

Notice that, according to (3.4)-(3.5), we have that $\boldsymbol{u}(t) \in \mathcal{U}$ at each $t \in[0, T]$. On the other hand, for all $\boldsymbol{w} \in \mathcal{U}$ there exists a unique $W \in \mathcal{W}$ such that $\boldsymbol{n} \times \boldsymbol{w} \times \boldsymbol{n}=-\operatorname{grad}_{\tau} W$ on $\partial \Omega$, where $\mathcal{W}$ is defined by

$$
\mathcal{W}:=\left\{W \in \mathrm{H}^{1 / 2}(\partial \Omega) / \mathbb{R}:\left.W\right|_{\Gamma_{\mathrm{J}}^{n}} \text { and }\left.W\right|_{\Gamma_{\mathrm{E}}^{n}} \text { constant, } n=1, \ldots, N\right\}
$$

(see Lem. 2.1 in [11]).

Let $L_{n}: \mathcal{U} \rightarrow \mathbb{R}, n=1, \ldots, N$, be defined by

$$
L_{n}(\boldsymbol{w}):=\left.W\right|_{\Gamma_{\mathrm{E}}^{n}}-\left.W\right|_{\Gamma_{\mathrm{J}}^{n}},
$$

where $W \in \mathcal{W}$ is the only function in this space such that $\boldsymbol{n} \times \boldsymbol{w} \times \boldsymbol{n}=-\operatorname{grad}_{\tau} W$ on $\partial \Omega$. We have that $L_{n}$ are bounded linear functionals. In fact,

$$
\left|L_{n}(\boldsymbol{w})\right| \leq \frac{1}{\left|\Gamma_{\mathrm{E}}^{n}\right|^{1 / 2}}\|W\|_{\mathrm{L}^{2}\left(\Gamma_{\mathrm{E}}^{n}\right)}+\frac{1}{\left|\Gamma_{\mathrm{J}}^{n}\right|^{1 / 2}}\|W\|_{\mathrm{L}^{2}\left(\Gamma_{\mathrm{J}}^{n}\right)} \leq C\|W\|_{\mathrm{H}^{1 / 2}(\partial \Omega)} \leq C\|\boldsymbol{w}\|_{\mathrm{H}(\mathbf{c u r l} ; \Omega)},
$$

where, for the last inequality, we have used results from [14], Remark 5.2. Here and thereafter $C$ denotes a generic constant not necessarily the same at each occurrence.

The following lemma will be used to impose the boundary conditions involving the input current intensities. Here and thereafter $\langle\cdot, \cdot\rangle$ denotes the duality pairing $\langle\cdot, \cdot\rangle_{\mathrm{H}_{\partial \Omega}^{-1 / 2}\left(\operatorname{div}_{\tau} ; \partial \Omega\right) \times \mathrm{H}_{\partial \Omega}^{-1 / 2}\left(\operatorname{curl}_{\tau} ; \partial \Omega\right)}$ as defined in Section 5 from [14].

Lemma 3.1. For all $\boldsymbol{G} \in \mathcal{X}$ and $W \in \mathcal{W}$ we have

$$
\left\langle\boldsymbol{G} \times \boldsymbol{n}, \operatorname{grad}_{\tau} W\right\rangle=\sum_{n=1}^{N}\left(\left.W\right|_{\Gamma_{\mathrm{E}}^{n}}-\left.W\right|_{\Gamma_{\mathrm{J}}^{n}}\right)\langle\operatorname{curl} \boldsymbol{G} \cdot \boldsymbol{n}, 1\rangle_{\Gamma_{\mathrm{J}}^{n}}
$$

Proof. Let $\Phi_{n}$ be any smooth function defined in $\Omega$ and such that $\left.\Phi_{n}\right|_{\Omega_{\mathrm{C}}^{m}}=\delta_{n m}, n=1, \ldots, N, m=1, \ldots, M$. Then, for $\boldsymbol{G} \in \mathcal{X}$,

$$
\langle\operatorname{curl} \boldsymbol{G} \cdot \boldsymbol{n}, 1\rangle_{\Gamma_{\mathrm{J}}^{n}}+\langle\operatorname{curl} \boldsymbol{G} \cdot \boldsymbol{n}, 1\rangle_{\Gamma_{\mathrm{E}}^{n}}=\left\langle\operatorname{curl} \boldsymbol{G} \cdot \boldsymbol{n}, \Phi_{n}\right\rangle_{\partial \Omega}=\int_{\Omega} \operatorname{curl} \boldsymbol{G} \cdot \operatorname{grad} \Phi_{n}=\int_{\Omega_{\mathrm{C}}^{n}} \operatorname{curl} \boldsymbol{G} \cdot \operatorname{grad} \Phi_{n}=0
$$

Hence, $\langle\operatorname{curl} \boldsymbol{G} \cdot \boldsymbol{n}, 1\rangle_{\Gamma_{\mathrm{J}}^{n}}=-\langle\operatorname{curl} \boldsymbol{G} \cdot \boldsymbol{n}, 1\rangle_{\Gamma_{\mathrm{E}}^{n}}, n=1, \ldots, N$. 
Therefore, for $W \in \mathcal{W}$, if $\Psi \in \mathrm{H}^{1}(\Omega)$ is such that $\left.\Psi\right|_{\partial \Omega}=W$

$$
\begin{aligned}
\left\langle\boldsymbol{G} \times \boldsymbol{n}, \operatorname{grad}_{\tau} W\right\rangle & =-\int_{\Omega} \operatorname{curl} \boldsymbol{G} \cdot \operatorname{grad} \Psi=-\langle\operatorname{curl} \boldsymbol{G} \cdot \boldsymbol{n}, W\rangle_{\partial \Omega} \\
& =-\left.\sum_{n=1}^{N} W\right|_{\Gamma_{\mathrm{J}}^{n}}\langle\operatorname{curl} \boldsymbol{G} \cdot \boldsymbol{n}, 1\rangle_{\Gamma_{\mathrm{J}}^{n}}-\left.\sum_{n=1}^{N} W\right|_{\Gamma_{\mathrm{E}}^{n}}\langle\operatorname{curl} \boldsymbol{G} \cdot \boldsymbol{n}, 1\rangle_{\Gamma_{\mathrm{E}}^{n}} \\
& =\sum_{n=1}^{N}\left(\left.W\right|_{\Gamma_{\mathrm{E}}^{n}}-\left.W\right|_{\Gamma_{\mathrm{J}}^{n}}\right)\langle\operatorname{curl} \boldsymbol{G} \cdot \boldsymbol{n}, 1\rangle_{\Gamma_{\mathrm{J}}^{n}} .
\end{aligned}
$$

Thus we conclude the proof.

Now, we are in a position to obtain a weak formulation of (3.3)-(3.10). By testing (3.3) with $\boldsymbol{w} \in \mathcal{U}$ we have

$$
\int_{\Omega} \sigma \partial_{t} \boldsymbol{u} \cdot \boldsymbol{w}+\int_{\Omega} \frac{1}{\mu} \operatorname{curl} \boldsymbol{u} \cdot \operatorname{curl} \boldsymbol{w}-\left\langle\frac{1}{\mu} \operatorname{curl} \boldsymbol{u} \times \boldsymbol{n}, \boldsymbol{w}\right\rangle=\int_{\Omega} \operatorname{curl} \boldsymbol{H}_{0} \cdot \boldsymbol{w} .
$$

Let $W \in \mathcal{W}$ be such that $\boldsymbol{n} \times \boldsymbol{w} \times \boldsymbol{n}=-\operatorname{grad}_{\tau} W$ on $\partial \Omega$. Provided $\boldsymbol{H} \in \mathcal{X}$, according to $(3.2), \frac{1}{\mu} \operatorname{curl} \boldsymbol{u}(t) \in \mathcal{X}$. Then, applying Lemma 3.1 we have that

$$
\begin{aligned}
\left\langle\frac{1}{\mu} \operatorname{curl} \boldsymbol{u}(t) \times \boldsymbol{n}, \boldsymbol{w}\right\rangle & =-\left\langle\frac{1}{\mu} \operatorname{curl} \boldsymbol{u}(t) \times \boldsymbol{n}, \operatorname{grad}_{\tau} W\right\rangle \\
& =-\sum_{n=1}^{N}\left(\left.W\right|_{\Gamma_{\mathrm{E}}^{n}}-\left.W\right|_{\Gamma_{\mathrm{J}}^{n}}\right)\left\langle\operatorname{curl}\left(\frac{1}{\mu} \operatorname{curl} \boldsymbol{u}(t)\right) \cdot \boldsymbol{n}, 1\right\rangle_{\Gamma_{\mathrm{J}}^{n}} \\
& =\sum_{n=1}^{N} L_{n}(\boldsymbol{w})\left\langle\left(\sigma \partial_{t} \boldsymbol{u}(t)-\operatorname{curl} \boldsymbol{H}_{0}\right) \cdot \boldsymbol{n}, 1\right\rangle_{\Gamma_{\mathrm{J}}^{n}} \\
& =\sum_{n=1}^{N} L_{n}(\boldsymbol{w})\left(I_{n}(t)-I_{n}(0)\right),
\end{aligned}
$$

the last equality because of (3.3), (3.1) and (3.9).

Therefore

$$
\int_{\Omega_{\mathrm{C}}} \sigma \partial_{t} \boldsymbol{u}(t) \cdot \boldsymbol{w}+\int_{\Omega} \frac{1}{\mu} \operatorname{curl} \boldsymbol{u}(t) \cdot \operatorname{curl} \boldsymbol{w}=\sum_{n=1}^{N} L_{n}(\boldsymbol{w})\left(I_{n}(t)-I_{n}(0)\right)+\int_{\Omega} \operatorname{curl} \boldsymbol{H}_{0} \cdot \boldsymbol{w} \quad \forall \boldsymbol{w} \in \mathcal{U} .
$$

On the other hand, we introduce the following space to impose (3.6)-(3.8) by means of a Lagrange multiplier:

$$
\mathcal{M}\left(\Omega_{\mathrm{D}}\right):=\left\{\varphi \in \mathrm{H}^{1}\left(\Omega_{\mathrm{D}}\right):\left.\varphi\right|_{\Gamma_{\mathrm{I}}^{1}}=0,\left.\varphi\right|_{\Gamma_{\mathrm{I}}^{k}}=\text { constant }, k=2, \ldots, M\right\} .
$$

It is easy to show that, for $\boldsymbol{u}(t) \in \mathcal{U}$,

$$
\int_{\Omega_{\mathrm{D}}} \epsilon \boldsymbol{u}(t) \cdot \operatorname{grad} \varphi=\int_{\Gamma_{\mathrm{D}}}\left(\int_{0}^{t} g(s) \mathrm{d} s\right) \varphi \quad \forall \varphi \in \mathcal{M}\left(\Omega_{\mathrm{D}}\right) \Leftrightarrow \begin{cases}\operatorname{div}(\epsilon \boldsymbol{u}(t))=0 & \text { in } \Omega_{\mathrm{D}}, \\ \epsilon \boldsymbol{u}(t) \cdot \boldsymbol{n}=\int_{0}^{t} g(s) \mathrm{d} s & \text { on } \Gamma_{\mathrm{D}}, \\ \langle\epsilon \boldsymbol{u}(t) \cdot \boldsymbol{n}, 1\rangle_{\Gamma_{\mathrm{I}}^{k}}=0, & k=2, \ldots, M .\end{cases}
$$


Thus, we are led to the following problem:

Problem 3.2. Given $g \in \mathrm{L}^{2}\left(0, T ; L^{2}\left(\Gamma_{\mathrm{D}}\right)\right), I_{n} \in \mathrm{H}^{2}(0, T), n=1, \ldots, N$, and $\boldsymbol{H}_{0}$ satisfying (3.1), find $\boldsymbol{u} \in$ $\mathrm{L}^{2}(0, T ; \mathcal{U})$ with $\left.\boldsymbol{u}\right|_{\Omega_{\mathrm{C}}} \in \mathrm{H}^{1}\left(0, T ; \mathrm{H}_{\Gamma_{\mathrm{C}}}\left(\operatorname{curl} ; \Omega_{\mathrm{C}}\right)\right)$ and $\xi \in \mathrm{L}^{2}\left(0, T ; \mathcal{M}\left(\Omega_{\mathrm{D}}\right)\right)$ such that

$$
\begin{aligned}
\int_{\Omega_{\mathrm{C}}} \sigma \partial_{t} \boldsymbol{u}(t) \cdot \boldsymbol{w}+\int_{\Omega} \frac{1}{\mu} \operatorname{curl} \boldsymbol{u}(t) \cdot \operatorname{curl} \boldsymbol{w} & +\int_{\Omega_{\mathrm{D}}} \epsilon \boldsymbol{w} \cdot \operatorname{grad} \xi(t) \\
& =\sum_{n=1}^{N} L_{n}(\boldsymbol{w})\left(I_{n}(t)-I_{n}(0)\right)+\int_{\Omega} \operatorname{curl} \boldsymbol{H}_{0} \cdot \boldsymbol{w} \quad \forall \boldsymbol{w} \in \mathcal{U}, \\
\int_{\Omega_{\mathrm{D}}} \epsilon \boldsymbol{u}(t) \cdot \operatorname{grad} \varphi=\int_{\Gamma_{\mathrm{D}}}\left(\int_{0}^{t} g(s) \mathrm{d} s\right) \varphi & \forall \varphi \in \mathcal{M}\left(\Omega_{\mathrm{D}}\right), \\
\boldsymbol{u}(0)=\mathbf{0} \quad \text { in } \Omega . &
\end{aligned}
$$

As stated above, an alternative weak formulation of (2.1)-(2.8) in terms of the magnetic field was analyzed in [9]. In this reference it was shown (cf. [9], Thm. 3.6) that there exists a unique $\boldsymbol{H} \in \mathrm{L}^{2}(0, T ; \mathcal{X}) \cap \mathrm{H}^{1}(0, T ; \mathcal{H} \boldsymbol{x})$ such that

$$
\begin{gathered}
\langle\operatorname{curl} \boldsymbol{H}(t) \cdot \boldsymbol{n}, 1\rangle_{\Gamma_{\mathrm{J}}^{n}}=I_{n}(t), \quad n=1, \ldots, N, \\
\int_{\Omega} \mu \partial_{t} \boldsymbol{H}(t) \cdot \boldsymbol{G}+\int_{\Omega_{\mathrm{C}}} \frac{1}{\sigma} \operatorname{curl} \boldsymbol{H}(t) \cdot \operatorname{curl} \boldsymbol{G}=0 \quad \forall \boldsymbol{G} \in \mathcal{V}, \\
\boldsymbol{H}(0)=\boldsymbol{H}_{0},
\end{gathered}
$$

where

$$
\mathcal{H}_{\boldsymbol{X}}:=\left\{\boldsymbol{G} \in \mathrm{L}^{2}(\Omega)^{3}: \operatorname{curl} \boldsymbol{G}=\mathbf{0} \text { in } \Omega_{\mathrm{D}}\right\} \quad \text { and } \quad \mathcal{V}:=\left\{\boldsymbol{G} \in \mathcal{X}:\langle\operatorname{curl} \boldsymbol{G} \cdot \boldsymbol{n}, 1\rangle_{\Gamma_{\mathrm{J}}^{n}}=0, n=1, \ldots, N\right\} .
$$

Moreover, it was shown in Theorem 3.8 of the same reference that defining $\boldsymbol{E}_{\mathrm{C}}(t):=\frac{1}{\sigma} \operatorname{curl} \boldsymbol{H}(t)$ in $\Omega_{\mathrm{C}}$, the following properties hold true a.e. $t \in(0, T)$ :

$$
\begin{aligned}
\operatorname{div}(\mu \boldsymbol{H}(t))=0 & \text { in } \Omega, \\
\mu \partial_{t} \boldsymbol{H}(t)+\operatorname{curl} \boldsymbol{E}_{\mathrm{C}}(t)=\mathbf{0} & \text { in } \Omega_{\mathrm{C}}, \\
\operatorname{curl} \boldsymbol{H}(t)=\mathbf{0} & \text { in } \Omega_{\mathrm{D}}, \\
\mu \boldsymbol{H}(t) \cdot \boldsymbol{n}=0 & \text { on } \partial \Omega, \\
\boldsymbol{E}_{\mathrm{C}}(t) \times \boldsymbol{n}=\mathbf{0} & \text { on } \Gamma_{\mathrm{C}}, \\
\langle\operatorname{curl} \boldsymbol{H}(t) \cdot \boldsymbol{n}, 1\rangle_{\Gamma_{\mathrm{J}}^{n}}=I_{n}(t), & n=1, \ldots, N .
\end{aligned}
$$

We will use these results to prove that Problem 3.2 also has a unique solution. With this aim, we need to extend $\boldsymbol{E}_{\mathrm{C}}$ to the dielectric domain in order to define $\boldsymbol{u}$ as its primitive. Next result shows how this can be done, taking into account that $\partial_{t} \boldsymbol{H} \in \mathrm{L}^{2}\left(0, T ; \mathrm{L}^{2}(\Omega)^{3}\right), \boldsymbol{E}_{\mathrm{C}} \in \mathrm{L}^{2}\left(0, T ; \mathrm{H}_{\Gamma_{\mathrm{C}}}\left(\mathbf{c u r l} ; \Omega_{\mathrm{C}}\right)\right)$ and $g \in \mathrm{L}^{2}\left(0, T ; \mathrm{L}^{2}\left(\Gamma_{\mathrm{D}}\right)\right)$.

Lemma 3.3. There exists a unique $\boldsymbol{E}_{\mathrm{D}} \in \mathrm{L}^{2}\left(0, T ; \mathrm{H}\left(\mathbf{c u r l} ; \Omega_{\mathrm{D}}\right)\right)$ which satisfies a.e. $t \in[0, T]$ :

$$
\begin{aligned}
\operatorname{curl} \boldsymbol{E}_{\mathrm{D}}(t)=-\mu \partial_{t} \boldsymbol{H}(t) & \text { in } \Omega_{\mathrm{D}}, \\
\boldsymbol{E}_{\mathrm{D}}(t) \times \boldsymbol{n}_{\mathrm{D}}=-\boldsymbol{E}_{\mathrm{C}}(t) \times \boldsymbol{n}_{\mathrm{C}} & \text { on } \Gamma_{\mathrm{I}}, \\
\operatorname{div}\left(\epsilon \boldsymbol{E}_{\mathrm{D}}(t)\right)=0 & \text { in } \Omega_{\mathrm{D}}, \\
\epsilon \boldsymbol{E}_{\mathrm{D}}(t) \cdot \boldsymbol{n}=g(t) & \text { on } \Gamma_{\mathrm{D}}, \\
\left\langle\epsilon \boldsymbol{E}_{\mathrm{D}}(t) \cdot \boldsymbol{n}, 1\right\rangle_{\Gamma_{\mathrm{I}}^{k}}=0, & k=2, \ldots, M .
\end{aligned}
$$


Proof. To prove that this problem is well-posed, let us write $\boldsymbol{E}_{\mathrm{D}}(t):=\widetilde{\boldsymbol{E}}_{\mathrm{D}}(t)+\widehat{\boldsymbol{E}}_{\mathrm{D}}(t)$ a.e. $t \in[0, T]$, where $\widetilde{\boldsymbol{E}}_{\mathrm{D}}(t), \widehat{\boldsymbol{E}}_{\mathrm{D}}(t) \in \mathrm{H}\left(\mathbf{c u r l} ; \Omega_{\mathrm{D}}\right)$ are respective solutions to the following problems:

$$
\begin{aligned}
\operatorname{curl} \widetilde{\boldsymbol{E}}_{\mathrm{D}}(t)=\mathbf{0} & \text { in } \Omega_{\mathrm{D}}, \\
\widetilde{\boldsymbol{E}}_{\mathrm{D}}(t) \times \boldsymbol{n}_{\mathrm{D}}=\mathbf{0} & \text { on } \Gamma_{\mathrm{I}}, \\
\operatorname{div}\left(\epsilon \widetilde{\boldsymbol{E}}_{\mathrm{D}}(t)\right)=0 & \text { in } \Omega_{\mathrm{D}}, \\
\epsilon \widetilde{\boldsymbol{E}}_{\mathrm{D}}(t) \cdot \boldsymbol{n}=g(t) & \text { on } \Gamma_{\mathrm{D}}, \\
\left\langle\epsilon \widetilde{\boldsymbol{E}}_{\mathrm{D}}(t) \cdot \boldsymbol{n}, 1\right\rangle_{\Gamma_{\mathrm{I}}^{k}}=0, & k=2, \ldots, M,
\end{aligned}
$$

and

$$
\begin{aligned}
\operatorname{curl} \widehat{\boldsymbol{E}}_{\mathrm{D}}(t)=-\mu \partial_{t} \boldsymbol{H}(t) & \text { in } \Omega_{\mathrm{D}}, \\
\widehat{\boldsymbol{E}}_{\mathrm{D}}(t) \times \boldsymbol{n}_{\mathrm{D}}=-\boldsymbol{E}_{\mathrm{C}}(t) \times \boldsymbol{n}_{\mathrm{C}} & \text { on } \Gamma_{\mathrm{I}}, \\
\operatorname{div}\left(\epsilon \widehat{\boldsymbol{E}}_{\mathrm{D}}(t)\right)=0 & \text { in } \Omega_{\mathrm{D}}, \\
\epsilon \widehat{\boldsymbol{E}}_{\mathrm{D}}(t) \cdot \boldsymbol{n}=0 & \text { on } \Gamma_{\mathrm{D}}, \\
\left\langle\epsilon \widehat{\boldsymbol{E}}_{\mathrm{D}}(t) \cdot \boldsymbol{n}, 1\right\rangle_{\Gamma_{\mathrm{I}}^{k}}=0, & k=2, \ldots, M .
\end{aligned}
$$

It was proved in [17], Theorem 8.4 (see also [18], Lem. 3.2) that for $g(t) \in \mathrm{L}^{2}\left(\Gamma_{\mathrm{D}}\right)$, the first problem has a unique solution $\widetilde{\boldsymbol{E}}_{\mathrm{D}}(t)$ which satisfies

$$
\left\|\widetilde{\boldsymbol{E}}_{\mathrm{D}}(t)\right\|_{\mathrm{H}\left(\mathbf{c u r l} ; \Omega_{\mathrm{D}}\right)} \leq C\|g(t)\|_{\mathrm{L}^{2}\left(\Gamma_{\mathrm{D}}\right)} .
$$

To prove that the second problem is also well-posed, we follow the steps of the proof of [5], Theorem 8.6, where a similar result was obtained in the harmonic case and for a particular topology. The key point of this proof is that the term $\mu \partial_{t} \boldsymbol{H}(t, \cdot) \in \mathrm{L}^{2}\left(\Omega_{\mathrm{D}}\right)^{3}$. Moreover, we also obtain

$$
\left\|\widehat{\boldsymbol{E}}_{\mathrm{D}}(t)\right\|_{\mathrm{H}\left(\mathbf{c u r l} ; \Omega_{\mathrm{D}}\right)} \leq C\left\{\left\|\partial_{t} \boldsymbol{H}(t)\right\|_{\mathrm{L}^{2}\left(\Omega_{\mathrm{D}}\right)^{3}}+\left\|\boldsymbol{E}_{\mathrm{C}}(t)\right\|_{\mathrm{H}\left(\mathbf{c u r l} ; \Omega_{\mathrm{C}}\right)}\right\} .
$$

Thus, we have that $\boldsymbol{E}_{\mathrm{D}}(t):=\widetilde{\boldsymbol{E}}_{\mathrm{D}}(t)+\widehat{\boldsymbol{E}}_{\mathrm{D}}(t)$ is a solution to problem (3.23)-(3.27). Furthermore, $\boldsymbol{E}_{\mathrm{D}} \in$ $\mathrm{L}^{2}\left(0, T ; \mathrm{H}\left(\mathbf{c u r l} ; \Omega_{\mathrm{D}}\right)\right)$ because of the above estimates for $\widetilde{\boldsymbol{E}}_{\mathrm{D}}$ and $\widehat{\boldsymbol{E}}_{\mathrm{D}}$. Moreover, this problem has at most one solution as a consequence of [17], Proposition 6.3. Thus, we conclude the proof.

Now, we are in a position to conclude the following result.

Theorem 3.4. Problem 3.2 has a unique solution $(\boldsymbol{u}, \xi)$, with Lagrange multiplier $\xi \equiv 0$.

Proof. To prove existence, let $\boldsymbol{H} \in \mathrm{L}^{2}(0, T ; \mathcal{X}) \cap \mathrm{H}^{1}\left(0, T ; \mathcal{H}_{\boldsymbol{x}}\right)$ be the solution of (3.14)-(3.16) and let $\boldsymbol{E}_{\mathrm{C}}:=$ $\left.\frac{1}{\sigma} \operatorname{curl} \boldsymbol{H}\right|_{\Omega_{\mathrm{C}}} \in \mathrm{L}^{2}\left(0, T ; \mathrm{H}_{\Gamma_{\mathrm{C}}}\left(\operatorname{curl} ; \Omega_{\mathrm{C}}\right)\right)$, so that $(3.17)-(3.22)$ hold true a.e. $t \in(0, T)$. Let

$$
\boldsymbol{E}(t):= \begin{cases}\boldsymbol{E}_{\mathrm{C}}(t) & \text { in } \Omega_{\mathrm{C}}, \\ \boldsymbol{E}_{\mathrm{D}}(t) & \text { in } \Omega_{\mathrm{D}}\end{cases}
$$

where $\boldsymbol{E}_{\mathrm{D}} \in \mathrm{L}^{2}\left(0, T ; \mathrm{H}\left(\mathbf{c u r l} ; \Omega_{\mathrm{D}}\right)\right)$ is the solution to $(3.23)-(3.27)$ a.e. $t \in(0, T)$. As a consequence of (3.24), $\boldsymbol{E}(t) \in \mathrm{H}(\operatorname{curl} ; \Omega)$ a.e. $t \in(0, T)$ and, hence $\boldsymbol{E} \in \mathrm{L}^{2}\left(0, T ; \mathrm{H}_{\Gamma_{\mathrm{C}}}(\right.$ curl $\left.; \Omega)\right)$. Thus, defining

$$
\boldsymbol{u}(t, \boldsymbol{x}):=\int_{0}^{t} \boldsymbol{E}(s, \boldsymbol{x}) \mathrm{d} s, \quad t \in[0, T], \quad \boldsymbol{x} \in \Omega,
$$


$\boldsymbol{u} \in \mathrm{L}^{2}\left(0, T ; \mathrm{H}_{\Gamma_{\mathrm{C}}}(\operatorname{curl} ; \Omega)\right)$, too. Moreover, from (3.18) and (3.23) we have that curl $\boldsymbol{E}=-\mu \partial_{t} \boldsymbol{H}$ in $\Omega$ and integrating in time

$$
\operatorname{curl} \boldsymbol{u}=\mu \boldsymbol{H}_{0}-\mu \boldsymbol{H} \quad \text { in }[0, T] \times \Omega .
$$

Therefore, from (3.1) and (3.20) we conclude that $\boldsymbol{u} \in \mathrm{L}^{2}(0, T ; \mathcal{U})$ and, since $\partial_{t} \boldsymbol{u}=\boldsymbol{E}$, we have that $\left.\boldsymbol{u}\right|_{\Omega_{\mathrm{C}}} \in$ $\mathrm{H}^{1}\left(0, T ; \mathrm{H}_{\Gamma_{\mathrm{C}}}\left(\operatorname{curl} ; \Omega_{\mathrm{C}}\right)\right)$.

Our next step is to prove that $(\boldsymbol{u}, 0)$ is a solution to Problem 3.2. With this aim, first we notice that by virtue of (3.17)-(3.22), the definition of $\boldsymbol{E}_{\mathrm{C}}(t)$ and (3.23)-(3.27), it is straightforward to show that $\boldsymbol{u}(t, \boldsymbol{x})$ satisfies (3.3)-(3.10). Then, the same steps that lead to (3.12) allow us to prove this expression in our case, which means that $(\boldsymbol{u}, 0)$ satisfies the first equation of Problem 3.2.

On the other hand, we integrate in time (3.25)-(3.27) and use the fact that $\boldsymbol{u}(0)=\mathbf{0}$ in $\Omega$, to conclude that $\boldsymbol{u}(t)$ satisfies the conditions on the right hand side of (3.13), which was shown to be equivalent to the second equation from Problem 3.2.

Thus, we have proved that $(\boldsymbol{u}, 0)$ is a solution of Problem 3.2. There only remains to prove that this problem has a unique solution. With this aim let $(\overline{\boldsymbol{u}}, \bar{\xi})$ be a solution of Problem 3.2 with vanishing data $g=0, I_{n}=0$, $n=1, \ldots, N$, and $\boldsymbol{H}_{0}=\mathbf{0}$, namely,

$$
\begin{aligned}
& \int_{\Omega_{\mathrm{C}}} \sigma \partial_{t} \overline{\boldsymbol{u}}(t) \cdot \boldsymbol{w}+\int_{\Omega} \frac{1}{\mu} \operatorname{curl} \overline{\boldsymbol{u}}(t) \cdot \operatorname{curl} \boldsymbol{w}+\int_{\Omega_{\mathrm{D}}} \epsilon \boldsymbol{w} \cdot \operatorname{grad} \bar{\xi}(t)=0 \quad \forall \boldsymbol{w} \in \mathcal{U}, \\
& \int_{\Omega_{\mathrm{D}}} \epsilon \overline{\boldsymbol{u}}(t) \cdot \operatorname{grad} \varphi=0 \quad \forall \varphi \in \mathcal{M}\left(\Omega_{\mathrm{D}}\right), \\
& \overline{\boldsymbol{u}}(0)=\mathbf{0} \quad \text { in } \Omega .
\end{aligned}
$$

By taking $\boldsymbol{w}=\overline{\boldsymbol{u}}(t)$ and $\varphi=\bar{\xi}(t)$, we obtain

$$
\frac{1}{2} \frac{\mathrm{d}}{\mathrm{d} t} \int_{\Omega_{\mathrm{C}}} \sigma|\overline{\boldsymbol{u}}(t)|^{2}+\int_{\Omega} \frac{1}{\mu}|\operatorname{curl} \overline{\boldsymbol{u}}(t)|^{2}=0 \quad \text { a.e. } t \in[0, T]
$$

and integrating in time

$$
\frac{1}{2} \underline{\sigma}\|\overline{\boldsymbol{u}}(t)\|_{\mathrm{L}^{2}\left(\Omega_{\mathrm{C}}\right)^{3}}^{2}+\int_{0}^{t} \int_{\Omega} \frac{1}{\mu}|\operatorname{curl} \overline{\boldsymbol{u}}(s)|^{2} \mathrm{~d} s \leq 0,
$$

which implies that $\overline{\boldsymbol{u}}(t)=\mathbf{0}$ in $\Omega_{\mathrm{C}}$ and $\operatorname{curl} \overline{\boldsymbol{u}}(t)=\mathbf{0}$ in $\Omega$. From this (3.32) and (3.13), we deduce that $\overline{\boldsymbol{u}}(t)$ is a solution of (3.23)-(3.27) with vanishing right hand sides. Hence $\overline{\boldsymbol{u}}(t) \equiv \mathbf{0}$ in $\Omega_{\mathrm{D}}$ (see Prop. 6.3 in [17]) and we conclude that $\overline{\boldsymbol{u}}(t)$ vanishes in the whole domain.

On the other hand, let $\widetilde{\xi}(t)$ be the extension of $\bar{\xi}(t)$ defined by: $\left.\widetilde{\xi}(t)\right|_{\Omega_{\mathrm{C}}^{k}}=\left.\bar{\xi}(t)\right|_{\Gamma_{\mathrm{I}}^{k}}, k=1, \ldots, M$. Then $\operatorname{grad} \widetilde{\xi}(t) \in \mathcal{U}$ and taking $\boldsymbol{w}=\operatorname{grad} \widetilde{\xi}(t)$ in (3.31) we obtain $\operatorname{grad} \bar{\xi}(t)=\mathbf{0}$ in $\Omega_{\mathrm{D}}$. Hence, $\bar{\xi}(t)$ vanishes because $\Omega_{\mathrm{D}}$ is connected and $\left.\bar{\xi}(t)\right|_{\Gamma_{\mathrm{I}}^{1}}=0$.

Remark 3.5. As was shown in the proof of the previous theorem, actually $\boldsymbol{u} \in \mathrm{H}^{1}\left(0, T ; \mathrm{H}_{\Gamma_{\mathrm{C}}}(\mathbf{c u r l} ; \Omega)\right)$. Then, the physical quantities can be recovered from (3.29) and (3.30) as follows:

$$
\widetilde{\boldsymbol{E}}:=\partial_{t} \boldsymbol{u} \text { and } \widetilde{\boldsymbol{H}}:=\boldsymbol{H}_{0}-\frac{1}{\mu} \operatorname{curl} \boldsymbol{u} .
$$

Different choices of the data $g$ lead to different solutions $\boldsymbol{u}$ to Problem 3.2. However only $\left.\widetilde{\boldsymbol{E}}\right|_{\Omega_{\mathrm{D}}}$ actually depends on $g$. In fact, we have shown in the proof of the theorem above that $\widetilde{\boldsymbol{H}}$ as defined above is the solution $\boldsymbol{H}$ to problem (3.14)-(3.16) (which does not depend on $g$ ) and $\left.\widetilde{\boldsymbol{E}}\right|_{\Omega_{\mathrm{C}}}=\left.\boldsymbol{E}\right|_{\Omega_{\mathrm{C}}}=\left.\frac{1}{\sigma} \operatorname{curl} \boldsymbol{H}\right|_{\Omega_{\mathrm{C}}}$. This is an important fact because, if we do not know the values of $\epsilon \boldsymbol{E} \cdot \boldsymbol{n}$ on $\Gamma_{\mathrm{D}}$ and we are not interested in computing $\boldsymbol{E}$ in the dielectric, then we can simply choose $g=0$ and compute the magnetic field $\boldsymbol{H}$ in $\Omega$ and the electric field $\boldsymbol{E}$ in $\Omega_{\mathrm{C}}$, which are typically the most relevant quantities in physical applications. 


\subsection{Space discretization}

From now on, we assume that $\Omega, \Omega_{\mathrm{C}}$ and $\Omega_{\mathrm{D}}$ are Lipschitz polyhedra and consider regular tetrahedral meshes $\mathcal{T}_{h}$ of $\Omega$, such that each element $K \in \mathcal{T}_{h}$ is contained either in $\Omega_{\mathrm{C}}$ or in $\Omega_{\mathrm{D}}$ ( $h$ stands as usual for the corresponding mesh-size). Therefore, $\mathcal{T}_{h}^{\Omega_{\mathrm{D}}}:=\left\{K \in \mathcal{T}_{h}: K \subset \Omega_{\mathrm{D}}\right\}$ is a mesh of $\Omega_{\mathrm{D}}$. We employ edge finite elements to approximate $\boldsymbol{u}$, more precisely, lowest-order Nédélec finite elements:

$$
\mathcal{N}_{h}(\Omega):=\left\{\boldsymbol{w}_{h} \in \mathrm{H}(\operatorname{curl} ; \Omega):\left.\boldsymbol{w}_{h}\right|_{K} \in \mathcal{N}(K) \forall K \in \mathcal{T}_{h}\right\},
$$

where, for each tetrahedron $K$,

$$
\mathcal{N}(K):=\left\{\boldsymbol{w}_{h} \in \mathbb{P}_{1}^{3}: \boldsymbol{w}_{h}(\boldsymbol{x})=\mathbf{a} \times \boldsymbol{x}+\mathbf{b}, \mathbf{a}, \mathbf{b} \in \mathbb{R}^{3}, \boldsymbol{x} \in K\right\} .
$$

On the other hand, we use standard finite elements for the Lagrange multiplier $\xi$ :

$$
\mathcal{L}_{h}\left(\Omega_{\mathrm{D}}\right):=\left\{\varphi_{h} \in \mathrm{H}^{1}\left(\Omega_{\mathrm{D}}\right):\left.\varphi_{h}\right|_{K} \in \mathbb{P}_{1}(K) \quad \forall K \in \mathcal{T}_{h}^{\Omega_{\mathrm{D}}}\right\} .
$$

We introduce the following discrete spaces:

$$
\begin{aligned}
& \mathcal{U}_{h}:=\left\{\boldsymbol{w}_{h} \in \mathcal{N}_{h}(\Omega): \boldsymbol{w}_{h} \times \boldsymbol{n}=\mathbf{0} \text { on } \Gamma_{\mathrm{C}} \text { and } \operatorname{curl} \boldsymbol{w}_{h} \cdot \boldsymbol{n}=0 \text { on } \partial \Omega\right\}, \\
& \mathcal{Q}_{h}:=\left\{\varphi_{h} \in \mathcal{L}_{h}\left(\Omega_{\mathrm{D}}\right):\left.\varphi_{h}\right|_{\Gamma_{\mathrm{I}}^{1}}=0,\left.\varphi_{h}\right|_{\Gamma_{\mathrm{I}}^{k}}=\text { constant, } k=2, \ldots, M\right\} .
\end{aligned}
$$

To discretize Problem 3.2, we consider a convenient way to compute the right hand side for the discrete test functions. Let

$$
\widetilde{L}_{n}\left(\boldsymbol{w}_{h}\right):=\int_{C_{n}} \boldsymbol{w}_{h} \cdot \boldsymbol{t} \quad \forall \boldsymbol{w}_{h} \in \mathcal{U}_{h}
$$

where $C_{n}$ is a simple curve on $\partial \Omega$ joining $\Gamma_{\mathrm{E}}^{n}$ with $\Gamma_{\mathrm{J}}^{n}, n=1, \ldots, N$, and $\boldsymbol{t}$ being a unit vector tangent to $C_{n}$. It is easy to see that $L_{n}\left(\boldsymbol{w}_{h}\right)=\widetilde{L}_{n}\left(\boldsymbol{w}_{h}\right)$ for all $\boldsymbol{w}_{h} \in \mathcal{U}_{h}(c f$. [11], Lem. 2.4).

Then, the space discretization of Problem 3.2 reads as follows:

Problem 3.6. Given $g \in \mathrm{L}^{2}\left(0, T ; L^{2}\left(\Gamma_{\mathrm{D}}\right)\right), I_{n} \in \mathrm{H}^{2}(0, T), n=1, \ldots, N$, and the initial condition $\boldsymbol{H}_{0}$ satisfying (3.1), find $\boldsymbol{u}_{h}:[0, T] \rightarrow \mathcal{U}_{h}$ and $\xi_{h}:[0, T] \rightarrow \mathcal{Q}_{h}$ such that

$$
\begin{aligned}
& \int_{\Omega_{\mathrm{C}}} \sigma \partial_{t} \boldsymbol{u}_{h}(t) \cdot \boldsymbol{w}_{h}+\int_{\Omega} \frac{1}{\mu} \operatorname{curl} \boldsymbol{u}_{h}(t) \cdot \operatorname{curl} \boldsymbol{w}_{h}+\int_{\Omega_{\mathrm{D}}} \epsilon \boldsymbol{w}_{h} \cdot \operatorname{grad} \xi_{h}(t) \\
& \quad=\sum_{n=1}^{N} \widetilde{L}_{n}\left(\boldsymbol{w}_{h}\right)\left(I_{n}(t)-I_{n}(0)\right)+\int_{\Omega} \operatorname{curl} \boldsymbol{H}_{0} \cdot \boldsymbol{w}_{h} \quad \forall \boldsymbol{w}_{h} \in \mathcal{U}_{h}, \\
& \int_{\Omega_{\mathrm{D}}} \epsilon \boldsymbol{u}_{h}(t) \cdot \operatorname{grad} \varphi_{h}=\int_{\Gamma_{\mathrm{D}}}\left(\int_{0}^{t} g(s) \mathrm{d} s\right) \varphi_{h} \quad \forall \varphi_{h} \in \mathcal{Q}_{h}, \\
& \boldsymbol{u}_{h}(0)=\mathbf{0} \quad \text { in } \Omega .
\end{aligned}
$$

To prove that this problem is well-posed we will use the discrete kernel

$$
\mathcal{K}_{h}:=\left\{\boldsymbol{w}_{h} \in \mathcal{U}_{h}: \int_{\Omega_{\mathrm{D}}} \epsilon \boldsymbol{w}_{h} \cdot \operatorname{grad} \varphi_{h}=0 \quad \forall \varphi_{h} \in \mathcal{Q}_{h}\right\}
$$

and the following inf-sup condition. 
Lemma 3.7. There exists $\beta>0$ (independent of $h$ ) such that

$$
\sup _{\substack{\boldsymbol{w}_{h} \in \mathcal{U}_{h} \\ \boldsymbol{w}_{h} \neq \mathbf{0}}} \frac{\int_{\Omega_{\mathrm{D}}} \epsilon \boldsymbol{w}_{h} \cdot \operatorname{grad} \varphi_{h}}{\left\|\boldsymbol{w}_{h}\right\|_{\mathrm{H}(\operatorname{curl} ; \Omega)}} \geq \beta\left\|\varphi_{h}\right\|_{\mathrm{H}^{1}\left(\Omega_{\mathrm{D}}\right)^{3}} \quad \forall \varphi_{h} \in \mathcal{Q}_{h} .
$$

Proof. For $\varphi_{h} \in \mathcal{Q}_{h}$ let $\tilde{\varphi}_{h}$ be its extension to $\Omega_{\mathrm{C}}$ defined by $\left.\tilde{\varphi}_{h}\right|_{\Omega_{\mathrm{C}}^{k}}=\left.\varphi_{h}\right|_{\Gamma_{\mathrm{I}}^{k}}$ (constant), $k=1, \ldots, M$. Then, $\operatorname{grad} \tilde{\varphi}_{h} \in \mathcal{U}_{h}$ and

$$
\sup _{\substack{\boldsymbol{w}_{h} \in \mathcal{U}_{h} \\ \boldsymbol{w}_{h} \neq \mathbf{0}}} \frac{\int_{\Omega_{\mathrm{D}}} \epsilon \boldsymbol{w}_{h} \cdot \operatorname{grad} \varphi_{h}}{\left\|\boldsymbol{w}_{h}\right\|_{\mathrm{H}(\operatorname{curl} ; \Omega)}} \geq \frac{\int_{\Omega_{\mathrm{D}}} \epsilon \operatorname{grad} \tilde{\varphi}_{h} \cdot \operatorname{grad} \varphi_{h}}{\left\|\operatorname{grad} \tilde{\varphi}_{h}\right\|_{\mathrm{H}(\operatorname{curl} ; \Omega)}} \geq \frac{\underline{\epsilon}\left\|\operatorname{grad} \varphi_{h}\right\|_{\mathrm{L}^{2}\left(\Omega_{\mathrm{D}}\right)^{3}}^{2}}{\left\|\operatorname{grad} \varphi_{h}\right\|_{\mathrm{L}^{2}\left(\Omega_{\mathrm{D}}\right)^{3}}} \geq \beta\left\|\varphi_{h}\right\|_{\mathrm{H}^{1}\left(\Omega_{\mathrm{D}}\right)^{3}},
$$

where we have used Poincaré inequality since, for all $\varphi_{h} \in \mathcal{Q}_{h},\left.\varphi_{h}\right|_{\Gamma_{\mathrm{I}}^{1}}=0$.

Next step is to prove that there exist a particular solution to equation (3.36).

Lemma 3.8. Given $g \in \mathrm{L}^{2}\left(0, T ; L^{2}\left(\Gamma_{\mathrm{D}}\right)\right)$, there exists $\widehat{\boldsymbol{u}}_{h} \in \mathrm{H}^{1}\left(0, T ; \mathcal{U}_{h}\right)$ such that

$$
\int_{\Omega_{\mathrm{D}}} \epsilon \widehat{\boldsymbol{u}}_{h}(t) \cdot \operatorname{grad} \varphi_{h}=\int_{\Gamma_{\mathrm{D}}}\left(\int_{0}^{t} g(s) \mathrm{d} s\right) \varphi_{h} \quad \forall \varphi_{h} \in \mathcal{Q}_{h} .
$$

Proof. Consider the following auxiliary problem: for each $t \in[0, T]$, find $\widehat{\boldsymbol{v}}_{h}(t) \in \mathcal{K}_{h}^{\perp \mathcal{u}_{h}}$ such that

$$
\int_{\Omega_{\mathrm{D}}} \epsilon \widehat{\boldsymbol{v}}_{h}(t) \cdot \operatorname{grad} \varphi_{h}=\int_{\Gamma_{\mathrm{D}}} g(t) \varphi_{h} \quad \forall \varphi_{h} \in \mathcal{Q}_{h} .
$$

According to [19], Lemma I.4.1 (iii) because of the inf-sup condition (3.38), this problem has a unique solution and the following estimate holds true:

$$
\left\|\widehat{\boldsymbol{v}}_{h}(t)\right\|_{\mathrm{H}(\mathbf{c u r l} ; \Omega)} \leq C\|g(t)\|_{\mathrm{L}^{2}\left(\Gamma_{\mathrm{D}}\right)}, \quad t \in[0, T] .
$$

Now, let $\widehat{\boldsymbol{u}}_{h}(t):=\int_{0}^{t} \widehat{\boldsymbol{v}}_{h}(s) d s$. From the above inequality it is immediate to show that $\widehat{\boldsymbol{u}}_{h} \in \mathrm{H}^{1}\left(0, T ; \mathcal{U}_{h}\right)$ and that it satisfies (3.39).

Now, if we write $\boldsymbol{u}_{h}=\widetilde{\boldsymbol{u}}_{h}+\widehat{\boldsymbol{u}}_{h}$, Problem 3.6 is equivalent to finding $\widetilde{\boldsymbol{u}}_{h}:[0, T] \rightarrow \mathcal{K}_{h}$ such that

$$
\begin{aligned}
& \int_{\Omega_{\mathrm{C}}} \sigma \partial_{t} \widetilde{\boldsymbol{u}}_{h}(t) \cdot \boldsymbol{w}_{h}+\int_{\Omega} \frac{1}{\mu} \operatorname{curl} \widetilde{\boldsymbol{u}}_{h}(t) \cdot \operatorname{curl} \boldsymbol{w}_{h} \\
& =\sum_{n=1}^{N} \widetilde{L}_{n}\left(\boldsymbol{w}_{h}\right)\left(I_{n}(t)-I_{n}(0)\right)+\int_{\Omega} \operatorname{curl} \boldsymbol{H}_{0} \cdot \boldsymbol{w}_{h}-\int_{\Omega_{\mathrm{C}}} \sigma \partial_{t} \widehat{\boldsymbol{u}}_{h}(t) \cdot \boldsymbol{w}_{h}-\int_{\Omega} \frac{1}{\mu} \operatorname{curl} \widehat{\boldsymbol{u}}_{h}(t) \cdot \operatorname{curl} \boldsymbol{w}_{h} \quad \forall \boldsymbol{w}_{h} \in \mathcal{K}_{h},
\end{aligned}
$$

$\widetilde{\boldsymbol{u}}_{h}(0)=\mathbf{0}$ in $\Omega$.

In what follows we prove that this problem has a unique solution.

Lemma 3.9. There exists a unique $\widetilde{\boldsymbol{u}}_{h} \in \mathrm{H}^{1}\left(0, T ; \mathcal{K}_{h}\right)$ solution of $(3.40)-(3.41)$.

Proof. Let $\left\{\boldsymbol{\Phi}_{i}\right\}_{i=1}^{K}$ be a basis of $\mathcal{K}_{h}$ such that the last functions furnish a basis $\left\{\boldsymbol{\Phi}_{i}\right\}_{i=K_{1}+1}^{K}$ of the subspace $\left\{\boldsymbol{w}_{h} \in \mathcal{K}_{h}: \boldsymbol{w}_{h}=\mathbf{0}\right.$ in $\left.\Omega_{\mathrm{D}}\right\}$. We write

$$
\widetilde{\boldsymbol{u}}_{h}(t, \boldsymbol{x})=\sum_{i=1}^{K} \alpha_{i}(t) \boldsymbol{\Phi}_{i}(\boldsymbol{x})
$$


Let $\boldsymbol{\alpha}(t):=\left(\alpha_{i}(t)\right)_{1 \leq i \leq K}$ and $\boldsymbol{b}(t):=\left(b_{i}(t)\right)_{1 \leq i \leq K}$, with

$$
b_{i}(t):=\sum_{n=1}^{N} \widetilde{L}_{n}\left(\boldsymbol{\Phi}_{i}\right)\left(I_{n}(t)-I_{n}(0)\right)+\int_{\Omega} \operatorname{curl} \boldsymbol{H}_{0} \cdot \boldsymbol{\Phi}_{i}-\int_{\Omega_{\mathrm{C}}} \sigma \partial_{t} \widehat{\boldsymbol{u}}_{h}(t) \cdot \boldsymbol{\Phi}_{i}-\int_{\Omega} \frac{1}{\mu} \operatorname{curl} \widehat{\boldsymbol{u}}_{h}(t) \cdot \operatorname{curl} \boldsymbol{\Phi}_{i} .
$$

We consider $\mathcal{M}:=\left(M_{i j}\right)_{1 \leq i, j \leq K}$ and $\mathcal{K}:=\left(K_{i j}\right)_{1 \leq i, j \leq K}$ given by

$$
M_{i j}:=\int_{\Omega_{\mathrm{C}}} \sigma \boldsymbol{\Phi}_{i} \cdot \boldsymbol{\Phi}_{j}, \quad K_{i j}:=\int_{\Omega} \frac{1}{\mu} \operatorname{curl} \boldsymbol{\Phi}_{i} \cdot \operatorname{curl} \boldsymbol{\Phi}_{j}, \quad 1 \leq i, j \leq K .
$$

Then, (3.40)-(3.41) reads as follows: Find $\boldsymbol{\alpha}:[0, T] \rightarrow \mathbb{R}^{K}$ such that

$$
\begin{aligned}
& \mathcal{M} \boldsymbol{\alpha}^{\prime}(t)+\mathcal{K} \boldsymbol{\alpha}(t)=\boldsymbol{b}(t), \\
& \boldsymbol{\alpha}(0)=\mathbf{0} .
\end{aligned}
$$

Because of the degenerate character of the problem, we decompose $\boldsymbol{\alpha}(t)$ as follows:

$$
\boldsymbol{\alpha}(t)=\left[\begin{array}{l}
\boldsymbol{\alpha}_{1}(t) \\
\boldsymbol{\alpha}_{2}(t)
\end{array}\right]
$$

with $\boldsymbol{\alpha}_{1}(t):=\left(\alpha_{i}(t)\right)_{1 \leq i \leq K_{1}}$. We use a similar decomposition for $\boldsymbol{b}(t)$ and matrices $\mathcal{M}$ and $\mathcal{K}$ to write

$$
\boldsymbol{b}(t)=\left[\begin{array}{l}
\boldsymbol{b}_{1}(t) \\
\boldsymbol{b}_{2}(t)
\end{array}\right], \quad \mathcal{M}=\left[\begin{array}{cc}
\mathcal{M}_{11} & \mathbf{0} \\
\mathbf{0} & \mathbf{0}
\end{array}\right], \quad \mathcal{K}=\left[\begin{array}{ll}
\mathcal{K}_{11} & \mathcal{K}_{12} \\
\mathcal{K}_{12}^{T} & \mathcal{K}_{22}
\end{array}\right]
$$

Provided $\mathcal{K}_{22}$ is invertible, (3.43) is equivalent to

$$
\begin{aligned}
& \mathcal{M}_{11} \boldsymbol{\alpha}_{1}^{\prime}(t)=\boldsymbol{b}_{1}(t)+\left[\mathcal{K}_{12} \mathcal{K}_{22}^{-1} \mathcal{K}_{12}^{T}-\mathcal{K}_{11}\right] \boldsymbol{\alpha}_{1}(t)-\mathcal{K}_{12} \mathcal{K}_{22}^{-1} \boldsymbol{b}_{2}(t) \\
& \boldsymbol{\alpha}_{1}(0)=\mathbf{0}
\end{aligned}
$$

Therefore, in such a case, the existence and uniqueness of solution of (3.43) follows from the fact that $\mathcal{M}_{11}$ is positive definite.

Thus, to conclude that (3.40)-(3.41) has a unique solution, we are going to check that $\mathcal{K}_{22}$ is positive definite. First notice that

$$
\boldsymbol{\beta}^{T} \mathcal{K}_{22} \boldsymbol{\beta}=\int_{\Omega_{\mathrm{D}}} \frac{1}{\mu}\left|\operatorname{curl}\left(\sum_{i=K_{1}+1}^{K} \beta_{i} \boldsymbol{\Phi}_{i}\right)\right|^{2} \geq 0
$$

Let us assume that the expression above vanishes. Then, $\boldsymbol{w}_{h}:=\sum_{i=K_{1}+1}^{K} \beta_{i} \boldsymbol{\Phi}_{i}$ satisfies

$$
\begin{aligned}
\boldsymbol{w}_{h} \in \mathcal{N}_{h}\left(\Omega_{\mathrm{D}}\right), & \\
\operatorname{curl} \boldsymbol{w}_{h}=\mathbf{0} & \text { in } \quad \Omega_{\mathrm{D}}, \\
\boldsymbol{w}_{h} \times \boldsymbol{n}=\mathbf{0} & \text { on } \quad \Gamma_{\mathrm{I}} \\
\int_{\Omega_{\mathrm{D}}} \epsilon \boldsymbol{w}_{h} \cdot \operatorname{grad} \varphi_{h}=0 & \forall \varphi_{h} \in \mathcal{Q}_{h} .
\end{aligned}
$$

Since $\Omega_{\mathrm{D}} \backslash \Sigma$ is pseudo Lipschitz and simply connected, as a consequence of (3.45) and (3.46) there exists $\vartheta_{h} \in \mathcal{L}_{h}\left(\Omega_{\mathrm{D}} \backslash \Sigma\right) / \mathbb{R}$ such that $\boldsymbol{w}_{h}=\widetilde{\operatorname{grad}} \vartheta_{h}$, where

$$
\mathcal{L}_{h}\left(\Omega_{\mathrm{D}} \backslash \Sigma\right):=\left\{\varrho_{h} \in \mathcal{C}\left(\Omega_{\mathrm{D}} \backslash \Sigma\right):\left.\varrho_{h}\right|_{K} \in \mathbb{P}_{1}(K) \quad \forall K \in \mathcal{T}_{h}^{\Omega_{\mathrm{D}}} \text { with } \llbracket \varrho_{h} \rrbracket_{\Sigma_{n}}=\text { constant, } n=1, \ldots, N\right\},
$$


with $\llbracket \cdot \rrbracket_{\Sigma_{n}}$ denoting the jump across $\Sigma_{n}$. From (3.47) we obtain $\operatorname{grad}_{\tau} \vartheta_{h}=\mathbf{0}$ on $\Gamma_{\mathrm{I}} \backslash \Sigma$. Thus, $\vartheta_{h}$ is constant on $\Gamma_{\mathrm{I}}^{n} \backslash \Sigma_{n}$, which implies that $\llbracket \vartheta_{h} \rrbracket_{\Sigma_{n}}=0, n=1, \ldots, N$, and, whence, $\vartheta_{h}$ can be extended to a continuous function in $\Omega_{\mathrm{D}}$. By setting $\left.\vartheta_{h}\right|_{\Gamma_{\mathrm{I}}^{1}}=0$ we obtain $\vartheta_{h} \in \mathcal{Q}_{h}$ and, from (3.48), $\vartheta_{h}$ is a constant in $\Omega_{\mathrm{D}}$ and then $\boldsymbol{w}_{h}=\mathbf{0}$ in $\Omega_{\mathrm{D}}$. Therefore, we conclude that $\mathcal{K}_{22}$ is positive definite.

Thus, we have shown that (3.43) has a unique solution $\boldsymbol{\alpha} \in \mathrm{H}^{1}\left(0, T ; \mathbb{R}^{K}\right)$ and, consequently, (3.40)-(3.41) also has a unique solution $\widetilde{\boldsymbol{u}}_{h} \in \mathrm{H}^{1}\left(0, T ; \mathcal{K}_{h}\right)$.

Finally, notice that for any solution to Problem 3.6, the Lagrange multiplier $\xi_{h}$ necessarily vanishes, as in the continuous case.

Lemma 3.10. If $\left(\boldsymbol{u}_{h}, \xi_{h}\right)$ is a solution to Problem 3.6, then $\xi_{h} \equiv 0$.

Proof. Let $\tilde{\xi}_{h}$ be extension to $\Omega_{\mathrm{C}}$ of $\xi_{h}$ defined by $\left.\tilde{\xi}_{h}\right|_{\Omega_{\mathrm{C}}^{k}}=\left.\xi_{h}\right|_{\Gamma_{\mathrm{I}}^{k}}, k=1, \ldots, M$. Then, grad $\tilde{\xi}_{h} \in \mathcal{U}_{h}$ and $\widetilde{L}_{n}\left(\operatorname{grad} \tilde{\xi}_{h}\right)=0, n=1, \ldots, N$. Furthermore, $\int_{\Omega_{\mathrm{C}}} \sigma \partial_{t} \boldsymbol{u}_{h} \cdot \operatorname{grad} \tilde{\xi}_{h}=0$ and $\int_{\Omega} \operatorname{curl} \boldsymbol{H}_{0} \cdot \operatorname{grad} \tilde{\xi}_{h}=0$, because $\operatorname{grad} \tilde{\xi}_{h}$ vanishes in $\Omega_{\mathrm{C}}$ and $\operatorname{curl} \boldsymbol{H}_{0}$ vanishes in $\Omega_{\mathrm{D}}$. Hence, taking $\boldsymbol{w}_{h}=\operatorname{grad} \tilde{\xi}_{h}$ in (3.35), we obtain that $\int_{\Omega_{\mathrm{D}}} \epsilon\left|\operatorname{grad} \xi_{h}\right|^{2}=0$. Therefore, $\xi_{h}$ is a constant in $\Omega_{\mathrm{D}}$ and, since $\left.\xi_{h}\right|_{\Gamma_{\mathrm{I}}^{1}}=0$, we have $\xi_{h} \equiv 0$.

Now, we are in a position to conclude the following result.

Theorem 3.11. Problem 3.6 has a unique solution $\left(\boldsymbol{u}_{h}, \xi_{h}\right)$, with $\boldsymbol{u}_{h} \in \mathrm{H}^{1}\left(0, T ; \mathcal{U}_{h}\right)$ and $\xi_{h}=0$.

Proof. Let $\boldsymbol{u}_{h}:=\widehat{\boldsymbol{u}}_{h}+\widetilde{\boldsymbol{u}}_{h}$, with $\widehat{\boldsymbol{u}}_{h}$ and $\widetilde{\boldsymbol{u}}_{h}$ being respective solutions of (3.39) and (3.40)-(3.41), then, for any $\xi_{h}:[0, T] \rightarrow \mathcal{Q}_{h},\left(\boldsymbol{u}_{h}, \xi_{h}\right)$ satisfies (3.35)-(3.37), the first equation only for $\boldsymbol{w}_{h} \in \mathcal{K}_{h}$. Hence, because of [19], Lemma I.4.1 (ii) and the inf-sup condition (3.38), for each $t \in[0, T]$ there exists a unique $\xi_{h}(t) \in \mathcal{Q}_{h}$ such that (3.35) holds for all $\boldsymbol{w}_{h} \in \mathcal{U}_{h}$. Moreover, according to Lemma 3.10, $\xi_{h}=0$. Finally, the uniqueness follows from the fact that $\left(\boldsymbol{u}_{h}, \xi_{h}\right)$ is a solution to Problem 3.6 if and only if $\widetilde{\boldsymbol{u}}_{h}=\boldsymbol{u}-\widehat{\boldsymbol{u}}_{h}$ is the unique solution to (3.40)-(3.41) (cf. Lem. 3.9) and $\xi_{h}=0$.

Our next goal is to obtain error estimates for this semi-discrete scheme. With this aim, from now on, we assume that the solution to Problem 3.2 satisfies $\boldsymbol{u} \in \mathrm{H}^{1}\left(0, T ; \mathrm{H}^{r}(\mathbf{c u r l} ; \Omega)\right)$ for $r \in\left(\frac{1}{2}, 1\right]$, where $\mathrm{H}^{r}(\operatorname{curl} ; \Omega):=\left\{\boldsymbol{G} \in \mathrm{H}^{r}(\Omega)^{3}: \operatorname{curl} \boldsymbol{G} \in \mathrm{H}^{r}(\Omega)^{3}\right\}$. Let $\mathcal{I}_{h}^{\mathcal{N}}$ denote the Nédélec interpolant operator. According to [11] Lemma 2.2, we have that if $\boldsymbol{w} \in \mathrm{H}^{r}(\mathbf{c u r l} ; \Omega) \cap \mathcal{U}$, then $\mathcal{I}_{h}^{\mathcal{N}} \boldsymbol{w} \in \mathcal{U}_{h}$. We decompose the error of $\boldsymbol{u}$ as follows

$$
\boldsymbol{u}(t)-\boldsymbol{u}_{h}(t)=\boldsymbol{\rho}_{h}(t)-\boldsymbol{\delta}_{h}(t)
$$

with

$$
\boldsymbol{\rho}_{h}(t):=\boldsymbol{u}(t)-\mathcal{I}_{h}^{\mathcal{N}} \boldsymbol{u}(t) \quad \text { and } \quad \boldsymbol{\delta}_{h}(t):=\mathcal{I}_{h}^{\mathcal{N}} \boldsymbol{u}(t)-\boldsymbol{u}_{h}(t) .
$$

First, we prove the following auxiliary error estimate.

Lemma 3.12. Let $\boldsymbol{u}$ be the solution to Problem 3.2 and $\boldsymbol{u}_{h}$ that to Problem 3.6. If $\boldsymbol{u} \in \mathrm{H}^{1}\left(0, T ; \mathrm{H}^{r}(\mathbf{c u r l} ; \Omega)\right)$ with $r \in\left(\frac{1}{2}, 1\right]$, then there exists a constant $C>0$, independent of $h$, such that

$$
\begin{aligned}
\sup _{0 \leq t \leq T}\left\|\boldsymbol{\delta}_{h}(t)\right\|_{\mathrm{L}^{2}\left(\Omega_{\mathrm{C}}\right)^{3}}^{2}+\sup _{0 \leq t \leq T}\left\|\operatorname{curl} \boldsymbol{\delta}_{h}(t)\right\|_{\mathrm{L}^{2}(\Omega)^{3}}^{2} & +\int_{0}^{T}\left\|\partial_{t} \boldsymbol{\delta}_{h}(t)\right\|_{\mathrm{L}^{2}\left(\Omega_{\mathrm{C}}\right)^{3}}^{2} \mathrm{~d} t \\
& \leq C\left\{\sup _{0 \leq t \leq T}\left\|\operatorname{curl} \boldsymbol{\rho}_{h}(t)\right\|_{\mathrm{L}^{2}(\Omega)^{3}}^{2}+\int_{0}^{T}\left\|\partial_{t} \boldsymbol{\rho}_{h}(t)\right\|_{\mathrm{H}(\operatorname{curl} ; \Omega)}^{2} \mathrm{~d} t\right\}
\end{aligned}
$$


Proof. Since $\xi=0$ and $\xi_{h}=0$ (cf. Thm. 3.4 and 3.11), subtracting the first equation in Problem 3.6 from that in Problem 3.2, we have

$$
\int_{\Omega_{\mathrm{C}}} \sigma \partial_{t}\left(\boldsymbol{u}(t)-\boldsymbol{u}_{h}(t)\right) \cdot \boldsymbol{w}_{h}+\int_{\Omega} \frac{1}{\mu} \operatorname{curl}\left(\boldsymbol{u}(t)-\boldsymbol{u}_{h}(t)\right) \cdot \operatorname{curl} \boldsymbol{w}_{h}=0 \quad \forall \boldsymbol{w}_{h} \in \mathcal{U}_{h} .
$$

On the other hand, the assumed regularity of $\boldsymbol{u}$ implies that $\partial_{t}\left(\mathcal{I}_{h}^{\mathcal{N}} \boldsymbol{u}(t)\right)=\mathcal{I}_{h}^{\mathcal{N}}\left(\partial_{t} \boldsymbol{u}(t)\right)$ a.e. $t \in[0, T]$ (see Thems. 111 and 113 from [27]). Then, taking successively $\boldsymbol{w}_{h}=\boldsymbol{\delta}_{h}(t)$ and $\boldsymbol{w}_{h}=\partial_{t} \boldsymbol{\delta}_{h}(t)$ and using the decomposition (3.49), the lemma follows by applying standard arguments for parabolic problems (see, for instance, [1], Lem. 5.7).

Now, we are in a position to prove the following error estimates.

Theorem 3.13. Let $\boldsymbol{u}$ be the solution to Problem 3.2 and $\boldsymbol{u}_{h}$ that to Problem 3.6. If $\boldsymbol{u} \in \mathrm{H}^{1}\left(0, T ; \mathrm{H}^{r}(\mathbf{c u r l} ; \Omega)\right)$ with $r \in\left(\frac{1}{2}, 1\right]$, then there exists a constant $C>0$, independent of $h$, such that

$$
\begin{aligned}
& \sup _{0 \leq t \leq T}\left\|\boldsymbol{u}(t)-\boldsymbol{u}_{h}(t)\right\|_{\mathrm{L}^{2}\left(\Omega_{\mathrm{C}}\right)^{3}}^{2}+\sup _{0 \leq t \leq T}\left\|\operatorname{curl} \boldsymbol{u}(t)-\operatorname{curl} \boldsymbol{u}_{h}(t)\right\|_{\mathrm{L}^{2}(\Omega)^{3}}^{2}+\int_{0}^{T}\left\|\partial_{t}\left(\boldsymbol{u}(t)-\boldsymbol{u}_{h}(t)\right)\right\|_{\mathrm{L}^{2}\left(\Omega_{\mathrm{C}}\right)^{3}}^{2} \mathrm{~d} t \\
& \leq C h^{2 r}\left\{\sup _{0 \leq t \leq T}\|\boldsymbol{u}(t)\|_{\mathrm{H}^{r}(\operatorname{curl} ; \Omega)}^{2}+\int_{0}^{T}\left\|\partial_{t} \boldsymbol{u}(t)\right\|_{\mathrm{H}^{r}(\mathbf{c u r l} ; \Omega)}^{2} \mathrm{~d} t\right\} \\
& \leq C h^{2 r}\|\boldsymbol{u}\|_{\mathrm{H}^{1}\left(0, T ; \mathrm{H}^{r}(\operatorname{curl} ; \Omega)\right)}^{2} .
\end{aligned}
$$

Proof. Classical estimates for the Nédélec interpolant lead to

$$
\left\|\boldsymbol{\rho}_{h}(t)\right\|_{\mathrm{H}(\mathbf{c u r l} ; \Omega)} \leq C h^{r}\|\boldsymbol{u}(t)\|_{\mathrm{H}^{r}(\mathbf{c u r l} ; \Omega)}, \quad\left\|\partial_{t} \boldsymbol{\rho}_{h}(t)\right\|_{\mathrm{H}(\mathbf{c u r l} ; \Omega)} \leq C h^{r}\left\|\partial_{t} \boldsymbol{u}(t)\right\|_{\mathrm{H}^{r}(\mathbf{c u r l} ; \Omega)} .
$$

Thus, the result follows from the decomposition (3.49) by using these estimates and the previous lemma.

Remark 3.14. This theorem allows us to obtain error estimates for the physical variables of interest in most applications, $\left.\boldsymbol{E}\right|_{\Omega_{\mathrm{C}}}$ and $\boldsymbol{H}$. For the first one, we define $\boldsymbol{E}_{h}(t, \boldsymbol{x}):=\partial_{t} \boldsymbol{u}_{h}(t, \boldsymbol{x})$ and we have the following error estimate:

$$
\int_{0}^{T}\left\|\boldsymbol{E}(t)-\boldsymbol{E}_{h}(t)\right\|_{\mathrm{L}^{2}\left(\Omega_{\mathrm{C}}\right)^{3}}^{2} \mathrm{~d} t \leq C h^{2 r}\|\boldsymbol{u}\|_{\mathrm{H}^{1}\left(0, T ; \mathrm{H}^{r}(\operatorname{curl} ; \Omega)\right)}^{2} .
$$

To approximate $\boldsymbol{H}$, we make use of $(3.2)$ and define $\boldsymbol{H}_{h}(t, \boldsymbol{x}):=\boldsymbol{H}_{0}(\boldsymbol{x})-\frac{1}{\mu} \operatorname{curl} \boldsymbol{u}_{h}(t, \boldsymbol{x})$. Then, we have

$$
\sup _{0 \leq t \leq T}\left\|\boldsymbol{H}(t)-\boldsymbol{H}_{h}(t)\right\|_{\mathrm{L}^{2}(\Omega)^{3}}^{2} \leq C h^{2 r}\|\boldsymbol{u}\|_{\mathrm{H}^{1}\left(0, T ; \mathrm{H}^{r}(\operatorname{curl} ; \Omega)\right)}^{2}
$$

Remark 3.15. The assumption $\boldsymbol{u} \in \mathrm{H}^{1}\left(0, T ; \mathrm{H}^{r}(\mathbf{c u r l} ; \Omega)\right)$ does not seem realistic when the magnetic permeability of conductor and dielectric are not the same ( $c f .(3.3))$. However, Theorem 3.13 holds true if this assumption is substituted by $\left.\boldsymbol{u}\right|_{\Omega_{\mathrm{C}}} \in \mathrm{H}^{1}\left(0, T ; \mathrm{H}^{r}\left(\operatorname{curl} ; \Omega_{\mathrm{C}}\right)\right)$ and $\left.\boldsymbol{u}\right|_{\Omega_{\mathrm{D}}} \in \mathrm{H}^{1}\left(0, T ; \mathrm{H}^{r}\left(\operatorname{curl} ; \Omega_{\mathrm{D}}\right)\right)$. 


\subsection{Time discretization}

We consider a uniform partition of $[0, T], t_{k}:=k \Delta t, k=0, \ldots, M$, with time step $\Delta t:=\frac{T}{M}$. A fully discrete approximation of Problem 3.2 by means of a backward Euler scheme reads as follows:

Problem 3.16. Find $\boldsymbol{u}_{h}^{m} \in \mathcal{U}_{h}$ and $\xi_{h}^{m} \in \mathcal{Q}_{h}, m=1, \ldots, M$, such that

$$
\begin{aligned}
& \int_{\Omega_{\mathrm{C}}} \sigma \frac{\boldsymbol{u}_{h}^{m}-\boldsymbol{u}_{h}^{m-1}}{\Delta t} \cdot \boldsymbol{w}_{h}+\int_{\Omega} \frac{1}{\mu} \operatorname{curl} \boldsymbol{u}_{h}^{m} \cdot \operatorname{curl} \boldsymbol{w}_{h}+\int_{\Omega_{\mathrm{D}}} \epsilon \boldsymbol{w}_{h} \cdot \operatorname{grad} \xi_{h}^{m} \\
& \quad=\sum_{n=1}^{N} \widetilde{L}_{n}\left(\boldsymbol{w}_{h}\right)\left(I_{n}\left(t_{m}\right)-I_{n}(0)\right)+\int_{\Omega} \operatorname{curl} \boldsymbol{H}_{0} \cdot \boldsymbol{w}_{h} \quad \forall \boldsymbol{w}_{h} \in \mathcal{U}_{h}, \\
& \int_{\Omega_{\mathrm{D}}} \epsilon \boldsymbol{u}_{h}^{m} \cdot \operatorname{grad} \varphi_{h}=\int_{\Gamma_{\mathrm{D}}}\left(\int_{0}^{t_{m}} g(s) \mathrm{d} s\right) \varphi_{h} \quad \forall \varphi_{h} \in \mathcal{Q}_{h}, \\
& \boldsymbol{u}_{h}^{0}=\mathbf{0} \quad \text { in } \Omega .
\end{aligned}
$$

We proceed as for the semi-discrete scheme. First, the same arguments allows us to show that any solution of Problem 3.16 satisfies $\xi_{h}^{m}=0, m=1, \ldots, M$. Secondly, let $\widehat{\boldsymbol{u}}_{h} \in \mathrm{H}^{1}\left(0, T ; \boldsymbol{U}_{h}\right)$ be as above so that it satisfies (3.39). Let $\widehat{\boldsymbol{u}}_{h}^{m}:=\widehat{\boldsymbol{u}}_{h}\left(t_{m}\right)$ and $\boldsymbol{u}_{h}^{m}=\widetilde{\boldsymbol{u}}_{h}^{m}+\widehat{\boldsymbol{u}}_{h}^{m}$. Then, it is clear that Problem 3.16 has a unique solution if only if there exist unique $\widetilde{\boldsymbol{u}}_{h}^{m} \in \mathcal{K}_{h}, m=1, \ldots, M$, such that

$$
\begin{aligned}
\int_{\Omega_{\mathrm{C}}} \sigma \widetilde{\boldsymbol{u}}_{h}^{m} \cdot \boldsymbol{w}_{h}+\Delta t \int_{\Omega} \frac{1}{\mu} \operatorname{curl} \widetilde{\boldsymbol{u}}_{h}^{m} \cdot \operatorname{curl} \boldsymbol{w}_{h}=\int_{\Omega_{\mathrm{C}}} \sigma \widetilde{\boldsymbol{u}}_{h}^{m-1} \cdot \boldsymbol{w}_{h}-\int_{\Omega_{\mathrm{C}}} \sigma\left(\widehat{\boldsymbol{u}}_{h}^{m}-\widehat{\boldsymbol{u}}_{h}^{m-1}\right) \cdot \boldsymbol{w}_{h} \\
\quad-\Delta t \int_{\Omega} \frac{1}{\mu} \operatorname{curl} \widehat{\boldsymbol{u}}_{h}^{m} \cdot \operatorname{curl} \boldsymbol{w}_{h}+\Delta t \sum_{n=1}^{N} \widetilde{L}_{n}\left(\boldsymbol{w}_{h}\right)\left(I_{n}\left(t_{m}\right)-I_{n}(0)\right)+\Delta t \int_{\Omega} \operatorname{curl} \boldsymbol{H}_{0} \cdot \boldsymbol{w}_{h} \quad \forall \boldsymbol{w}_{h} \in \mathcal{K}_{h}
\end{aligned}
$$

with $\widetilde{\boldsymbol{u}}_{h}^{0}=\mathbf{0}$.

To prove that this problem has a unique solution, we proceed as in the proof of Lemma 3.9. We write $\widetilde{\boldsymbol{u}}_{h}^{m}$ in the basis $\left\{\boldsymbol{\Phi}_{i}\right\}_{i=1}^{K}$ of $\mathcal{K}_{h}, \widetilde{\boldsymbol{u}}_{h}^{m}=\sum_{i=1}^{K} \alpha_{i}^{m} \boldsymbol{\Phi}_{i}$, and obtain the following matrix form of the problem above:

$$
\widetilde{\mathcal{M}} \boldsymbol{\alpha}^{m}=\mathcal{M} \boldsymbol{\alpha}^{m-1}+\Delta t \boldsymbol{b}^{m},
$$

with $\boldsymbol{b}^{m} \in \mathbb{R}^{K}$ beging the vector arising from the right hand side of the problem, $\mathcal{M}$ as in (3.42) and

$$
\widetilde{\mathcal{M}}:=\mathcal{M}+\Delta t \mathcal{K}=\left[\begin{array}{cc}
\mathcal{M}_{11}+\Delta t \mathcal{K}_{11} & \Delta t \mathcal{K}_{12} \\
\Delta t \mathcal{K}_{12}^{T} & \Delta t \mathcal{K}_{22}
\end{array}\right],
$$

where we have used the block matrices from (3.44). Since $\mathcal{K}$ is semi-positive definite and $\mathcal{M}_{11}$ and $\mathcal{K}_{22}$ are positive definite, it is easy to check that $\widetilde{\mathcal{M}}$ is also positive definite. Thus, we conclude that Problem 3.16 has a unique solution.

Our next goal is to obtain error estimates for this fully-discrete scheme. With this aim, we write

$$
\partial_{t} \boldsymbol{u}\left(t_{k}\right)-\frac{\boldsymbol{u}_{h}^{k}-\boldsymbol{u}_{h}^{k-1}}{\Delta t}=\frac{\boldsymbol{\rho}_{h}^{k}-\boldsymbol{\rho}_{h}^{k-1}}{\Delta t}+\frac{\boldsymbol{\delta}_{h}^{k}-\boldsymbol{\delta}_{h}^{k-1}}{\Delta t}-\boldsymbol{\tau}^{k}
$$

where

$$
\boldsymbol{\rho}_{h}^{k}:=\boldsymbol{u}\left(t_{k}\right)-\mathcal{I}_{h}^{\mathcal{N}} \boldsymbol{u}\left(t_{k}\right), \quad \boldsymbol{\delta}_{h}^{k}:=\mathcal{I}_{h}^{\mathcal{N}} \boldsymbol{u}\left(t_{k}\right)-\boldsymbol{u}_{h}^{k} \quad \text { and } \quad \boldsymbol{\tau}^{k}:=\frac{\boldsymbol{u}\left(t_{k}\right)-\boldsymbol{u}\left(t_{k-1}\right)}{\Delta t}-\partial_{t} \boldsymbol{u}\left(t_{k}\right)
$$


Lemma 3.17. Let $\boldsymbol{u}$ be the solution to Problem 3.2 and $\boldsymbol{u}_{h}^{k}, k=1, \ldots, M$, that to Problem 3.16. If $\boldsymbol{u} \in$ $\mathrm{H}^{1}\left(0, T ; \mathrm{H}^{r}(\mathbf{c u r l} ; \Omega)\right)$ with $r \in\left(\frac{1}{2}, 1\right]$, then there exists a constant $C>0$, independent of $h$ and $\Delta t$, such that

$$
\begin{aligned}
& \max _{1 \leq k \leq M}\left\|\boldsymbol{\delta}_{h}^{k}\right\|_{\mathrm{L}^{2}\left(\Omega_{\mathrm{C}}\right)^{3}}^{2}+\max _{1 \leq k \leq M} \| \operatorname{curl} \boldsymbol{\delta}_{h}^{k}\left\|_{\mathrm{L}^{2}(\Omega)^{3}}^{2}+\Delta t \sum_{k=1}^{M}\right\| \frac{\boldsymbol{\delta}_{h}^{k}-\boldsymbol{\delta}_{h}^{k-1}}{\Delta t} \|_{\mathrm{L}^{2}\left(\Omega_{\mathrm{C}}\right)^{3}}^{2} \\
& \leq C\left(\max _{1 \leq k \leq M}\left\|\operatorname{curl} \boldsymbol{\rho}_{h}^{k}\right\|_{\mathrm{L}^{2}(\Omega)^{3}}^{2}+\Delta t \sum_{k=1}^{M}\left\{\left\|\boldsymbol{\tau}^{k}\right\|_{\mathrm{L}^{2}\left(\Omega_{\mathrm{C}}\right)^{3}}^{2}+\left\|\frac{\boldsymbol{\rho}_{h}^{k}-\boldsymbol{\rho}_{h}^{k-1}}{\Delta t}\right\|_{\mathrm{L}^{2}\left(\Omega_{\mathrm{C}}\right)^{3}}^{2}\right\}\right) .
\end{aligned}
$$

Proof. Since $\xi=0$ and $\xi_{h}^{k}=0, k=1, \ldots, M$, subtracting the first equation in Problem 3.16 from that in Problem 3.2, we obtain

$$
\int_{\Omega_{\mathrm{C}}} \sigma\left(\partial_{t} \boldsymbol{u}\left(t_{k}\right)-\frac{\boldsymbol{u}_{h}^{k}-\boldsymbol{u}_{h}^{k-1}}{\Delta t}\right) \cdot \boldsymbol{w}_{h}+\int_{\Omega} \frac{1}{\mu} \operatorname{curl}\left(\boldsymbol{u}\left(t_{k}\right)-\boldsymbol{u}_{h}^{k}\right) \cdot \operatorname{curl} \boldsymbol{w}_{h}=0 \quad \forall \boldsymbol{w}_{h} \in \mathcal{U}_{h} .
$$

Then, using (3.52) and the fact that $\boldsymbol{u}\left(t_{k}\right)-\boldsymbol{u}_{h}^{k}=\boldsymbol{\delta}_{h}^{k}+\boldsymbol{\rho}_{h}^{k}$, the lemma follows from standard arguments for parabolic problems (see, for instance, [1], Lem. 6.1).

Now, we are in a position to write one of the main results of this paper.

Theorem 3.18. Let $\boldsymbol{u}$ be the solution to Problem 3.2 and $\boldsymbol{u}_{h}^{k}, k=1, \ldots, M$, that to Problem 3.16. If $\boldsymbol{u} \in \mathrm{H}^{1}\left(0, T ; \mathrm{H}^{r}(\mathbf{c u r l} ; \Omega)\right)$ for $r \in\left(\frac{1}{2}, 1\right]$, and $\left.\boldsymbol{u}\right|_{\Omega_{\mathrm{C}}} \in \mathrm{H}^{2}\left(0, T ; \mathrm{L}^{2}\left(\Omega_{\mathrm{C}}\right)^{3}\right)$, then there exists a constant $C>0$, independent of $h$ and $\Delta t$, such that

$$
\begin{aligned}
& \max _{1 \leq k \leq M}\left\|\boldsymbol{u}\left(t_{k}\right)-\boldsymbol{u}_{h}^{k}\right\|_{\mathrm{L}^{2}\left(\Omega_{\mathrm{C}}\right)^{3}}^{2}+\max _{1 \leq k \leq M}\left\|\operatorname{curl}\left(\boldsymbol{u}\left(t_{k}\right)-\boldsymbol{u}_{h}^{k}\right)\right\|_{\mathrm{L}^{2}(\Omega)^{3}}^{2}+\Delta t \sum_{k=1}^{M}\left\|\partial_{t} \boldsymbol{u}\left(t_{k}\right)-\frac{\boldsymbol{u}_{h}^{k}-\boldsymbol{u}_{h}^{k-1}}{\Delta t}\right\|_{\mathrm{L}^{2}\left(\Omega_{\mathrm{C}}\right)^{3}}^{2} \\
& \quad \leq C\left\{(\Delta t)^{2} \int_{0}^{T}\left\|\partial_{t t} \boldsymbol{u}(t)\right\|_{\mathrm{L}^{2}\left(\Omega_{\mathrm{C}}\right)^{3}}^{2} \mathrm{~d} t+h^{2 r} \sup _{0 \leq t \leq T}\|\boldsymbol{u}(t)\|_{\mathrm{H}^{r}(\operatorname{curl} ; \Omega)}^{2}+h^{2 r} \int_{0}^{T}\left\|\partial_{t} \boldsymbol{u}(t)\right\|_{\mathrm{H}^{r}(\mathbf{c u r l} ; \Omega)}^{2} \mathrm{~d} t\right\} \\
& \quad \leq C\left\{(\Delta t)^{2}\|\boldsymbol{u}\|_{\mathrm{H}^{2}\left(0, T ; \mathrm{L}^{2}\left(\Omega_{\mathrm{C}}\right)^{3}\right)}^{2}+h^{2 r}\|\boldsymbol{u}\|_{\mathrm{H}^{1}\left(0, T ; \mathrm{H}^{r}(\operatorname{curl} ; \Omega)\right)}^{2}\right\} .
\end{aligned}
$$

Proof. A Taylor expansion shows that

$$
\sum_{k=1}^{M}\left\|\boldsymbol{\tau}^{k}\right\|_{\mathrm{L}^{2}\left(\Omega_{\mathrm{C}}\right)^{3}}^{2}=\sum_{k=1}^{M}\left\|\frac{1}{\Delta t} \int_{t_{k-1}}^{t_{k}}\left(t_{k}-s\right) \partial_{t t} \boldsymbol{u}(s) \mathrm{d} s\right\|_{\mathrm{L}^{2}\left(\Omega_{\mathrm{C}}\right)^{3}}^{2} \leq \Delta t \int_{0}^{T}\left\|\partial_{t t} \boldsymbol{u}(t)\right\|_{\mathrm{L}^{2}\left(\Omega_{\mathrm{C}}\right)^{3}}^{2} \mathrm{~d} t .
$$

Moreover,

$$
\sum_{k=1}^{M}\left\|\frac{\boldsymbol{\rho}_{h}^{k}-\boldsymbol{\rho}_{h}^{k-1}}{\Delta t}\right\|_{\mathrm{L}^{2}\left(\Omega_{\mathrm{C}}\right)^{3}}^{2} \leq \frac{1}{\Delta t} \int_{0}^{T}\left\|\partial_{t} \boldsymbol{\rho}_{h}(t)\right\|_{\mathrm{L}^{2}\left(\Omega_{\mathrm{C}}\right)^{3}}^{2} \mathrm{~d} t
$$

Since $\boldsymbol{u}\left(t_{k}\right)-\boldsymbol{u}_{h}^{k}=\boldsymbol{\delta}_{h}^{k}+\boldsymbol{\rho}_{h}^{k}$, the result follows from (3.51), (3.52) and the previous lemma.

Remark 3.19. As in the semi-discrete scheme, if we approximate the electric field $\boldsymbol{E}$ and the magnetic field $\boldsymbol{H}$ at each time $t_{k}, k=1, \ldots, M$, by taking $\boldsymbol{E}_{h}^{k}:=\frac{\boldsymbol{u}_{h}^{k}-\boldsymbol{u}_{h}^{k-1}}{\Delta t}$ and $\boldsymbol{H}_{h}^{k}:=\boldsymbol{H}_{0}-\frac{1}{\mu} \operatorname{curl} \boldsymbol{u}_{h}^{k}$, respectively, then

$$
\begin{aligned}
& \Delta t \sum_{k=1}^{M}\left\|\boldsymbol{E}\left(t_{k}\right)-\boldsymbol{E}_{h}^{k}\right\|_{\mathrm{L}^{2}\left(\Omega_{\mathrm{C}}\right)^{3}}^{2} \leq C\left\{(\Delta t)^{2}\|\boldsymbol{u}\|_{\mathrm{H}^{2}\left(0, T ; \mathrm{L}^{2}\left(\Omega_{\mathrm{C}}\right)^{3}\right)}^{2}+h^{2 r}\|\boldsymbol{u}\|_{\mathrm{H}^{1}\left(0, T ; \mathrm{H}^{r}(\operatorname{curl} ; \Omega)\right)}^{2}\right\}, \\
& \left.\max _{1 \leq k \leq M} \| \boldsymbol{H}\left(t_{k}\right)-\boldsymbol{H}_{h}^{k}\right) \|_{\mathrm{L}^{2}(\Omega)^{3}}^{2} \leq C\left\{(\Delta t)^{2}\|\boldsymbol{u}\|_{\mathrm{H}^{2}\left(0, T ; \mathrm{L}^{2}\left(\Omega_{\mathrm{C}}\right)^{3}\right)}^{2}+h^{2 r}\|\boldsymbol{u}\|_{\mathrm{H}^{1}\left(0, T ; \mathrm{H}^{r}(\operatorname{curl} ; \Omega)\right)}^{2}\right\} .
\end{aligned}
$$


Remark 3.20. The same observation made in Remark 3.15 holds in this case.

\section{EDdY CURRENT PROBLEM WITH VOLTAGE DROPS AS BOUNDARY DATA}

The goal of this section is to analyze the transient eddy current problem with voltage drops as boundary data. We consider equations (2.1)-(2.7) together with (2.9), for $n=1, \ldots, N$, and (2.10)-(2.12). Notice that the only difference with respect the problem studied in the previous section is that (2.9) replaces (2.8). As in the previous section, we assume that the initial data $\boldsymbol{H}_{0}$ satisfies (3.1) and that $g \in \mathrm{L}^{2}\left(0, T ; L^{2}\left(\Gamma_{\mathrm{D}}\right)\right)$. Furthermore, we assume that $V_{n} \in \mathrm{H}^{1}(0, T), n=1, \ldots, N$.

Let $\boldsymbol{u}(t):=\int_{0}^{t} \boldsymbol{E}(s) \mathrm{d} s$ as above. Integrating in time (2.9), we have $\boldsymbol{n} \times \boldsymbol{u}(t) \times \boldsymbol{n}=-\int_{0}^{t} \operatorname{grad}_{\tau} V(s) \mathrm{d} s=$ $-\operatorname{grad}_{\tau}\left(\int_{0}^{t} V(s) \mathrm{d} s\right)$ on $\partial \Omega$. Thus, according to (3.11), we have that $L_{n}(\boldsymbol{u}(t))=\int_{0}^{t} V_{n}(s) \mathrm{d} s, n=1, \ldots, N$, $t \in[0, T]$.

Therefore, the transient eddy current problem with voltage drops as boundary data written in terms of $\boldsymbol{u}$ is given by equations (3.3)-(3.8) and

$$
L_{n}(\boldsymbol{u}(t))=\int_{0}^{t} V_{n}(s) \mathrm{d} s, \quad n=1, \ldots, N, \quad t \in[0, T]
$$

(the latter instead of (3.9)), with the initial condition (3.10).

Similar arguments to those used in Section 3 allow us to obtain the following problem:

Problem 4.1. Given $g \in \mathrm{L}^{2}\left(0, T ; L^{2}\left(\Gamma_{\mathrm{D}}\right)\right), V_{n} \in \mathrm{H}^{1}(0, T), n=1, \ldots, N$, and an initial condition $\boldsymbol{H}_{0}$ satisfying (3.1), find $\boldsymbol{u} \in \mathrm{L}^{2}(0, T ; \mathcal{U})$ with $\left.\boldsymbol{u}\right|_{\Omega_{\mathrm{C}}} \in \mathrm{H}^{1}\left(0, T ; \mathrm{H}_{\Gamma_{\mathrm{C}}}\left(\operatorname{curl} ; \Omega_{\mathrm{C}}\right)\right)$ and $\xi \in \mathrm{L}^{2}\left(0, T ; \mathcal{M}\left(\Omega_{\mathrm{D}}\right)\right)$ such that

$$
\begin{aligned}
& L_{n}(\boldsymbol{u}(t))=\int_{0}^{t} V_{n}(s) \mathrm{d} s, \quad n=1, \ldots, N, \quad \text { a.e. } t \in[0, T], \\
& \int_{\Omega_{\mathrm{C}}} \sigma \partial_{t} \boldsymbol{u}(t) \cdot \boldsymbol{w}+\int_{\Omega} \frac{1}{\mu} \operatorname{curl} \boldsymbol{u}(t) \cdot \operatorname{curl} \boldsymbol{w}+\int_{\Omega_{\mathrm{D}}} \epsilon \boldsymbol{w} \cdot \operatorname{grad} \xi(t)=\int_{\Omega} \operatorname{curl} \boldsymbol{H}_{0} \cdot \boldsymbol{w} \quad \forall \boldsymbol{w} \in \mathcal{U}_{0}, \\
& \int_{\Omega_{\mathrm{D}}} \epsilon \boldsymbol{u}(t) \cdot \operatorname{grad} \varphi=\int_{\Gamma_{\mathrm{D}}}\left(\int_{0}^{t} g(s) \mathrm{d} s\right) \varphi \quad \forall \varphi \in \mathcal{M}\left(\Omega_{\mathrm{D}}\right), \\
& \boldsymbol{u}(0)=\mathbf{0} \quad \operatorname{in} \Omega
\end{aligned}
$$

where $\mathcal{U}^{0}=\left\{\boldsymbol{w} \in \mathcal{U}: L_{n}(\boldsymbol{w})=0, n=1, \ldots, N\right\}$.

A formulation of the same problem in terms of the magnetic field $\boldsymbol{H}$ was analyzed in [9]. In particular, it was shown in this reference that equations (2.1)-(2.7) with voltage drops $V_{n}(t), n=1, \ldots, N$, as boundary data lead to a well-posed problem (cf. [9], Rem. 3.7) which consists of finding $\boldsymbol{H} \in \mathrm{L}^{2}(0, T ; \mathcal{X}) \cap \mathrm{H}^{1}(0, T ; \mathcal{H} \boldsymbol{x})$ such that

$$
\begin{aligned}
\int_{\Omega} \mu \partial_{t} \boldsymbol{H}(t) \cdot \boldsymbol{G}+\int_{\Omega_{\mathrm{C}}} \frac{1}{\sigma} \operatorname{curl} \boldsymbol{H}(t) \cdot \operatorname{curl} \boldsymbol{G} & =-\sum_{n=1}^{N} V_{n}(t)\langle\operatorname{curl} \boldsymbol{G} \cdot \boldsymbol{n}, 1\rangle_{\Gamma_{\mathrm{J}}^{n}} \quad \forall \boldsymbol{G} \in \mathcal{X}, \\
\boldsymbol{H}(0) & =\boldsymbol{H}_{0}
\end{aligned}
$$

Defining $\boldsymbol{E}_{\mathrm{C}}(t):=\frac{1}{\sigma} \operatorname{curl} \boldsymbol{H}(t)$ in $\Omega_{\mathrm{C}}$, the arguments from [9], Theorem 3.8 can be repeated to prove that $\boldsymbol{H}(t)$ and $\boldsymbol{E}_{\mathrm{C}}(t)$ satisfy $(3.17)-(3.21)$, a.e. $t \in(0, T)$. 
Theorem 4.2. Problem 4.1 has a unique solution $(\boldsymbol{u}, \xi)$ and the Lagrange multiplier $\xi$ vanishes.

Proof. The existence of solution follows by repeating the arguments of the proof of Theorem 3.4. In fact, now we begin with the solution $\boldsymbol{H}$ of (4.5)-(4.6) (instead of that of (3.14)-(3.16)). Repeating the steps of the proof of Theorem 3.4, we define $\boldsymbol{E}_{\mathrm{C}}(t):=\frac{1}{\sigma} \operatorname{curl} \boldsymbol{H}(t)$ in $\Omega_{\mathrm{C}}$ and show that $\boldsymbol{H}(t)$ and $\boldsymbol{E}_{\mathrm{C}}(t)$ satisfy $(3.17)-(3.21)$ a.e. $t \in(0, T)$. Next, we define $\boldsymbol{E}_{\mathrm{D}}(t), t \in[0, T]$, as the solution of $(3.23)-(3.27), \boldsymbol{E}(t)$ as in (3.28) and $\boldsymbol{u}$ as in (3.29). Proceeding as in the proof of Theorem 3.4 and using the fact that $L_{n}(\boldsymbol{w})=0$ for $\boldsymbol{w} \in \mathcal{U}^{0}$, we prove that $(\boldsymbol{u}, 0)$ satisfies (4.2)-(4.4). Thus, to conclude the existence of solution, there only remains to prove that $\boldsymbol{u}$ satisfies (4.1).

To prove this, note that as a consequence of (3.18), (3.23), (3.24) and (3.20), there exists a function $\widetilde{V}$ defined in $\Omega$ up to a constant, such that $\left.\widetilde{V}\right|_{\partial \Omega}$ is a surface potential of the tangential component of $\boldsymbol{E}$; namely, $\boldsymbol{n} \times \boldsymbol{E} \times \boldsymbol{n}=-\operatorname{grad}_{\tau} \widetilde{V}$ on $\partial \Omega$. On the other hand, (3.21) implies that $\widetilde{V}$ is constant on each connected component of $\Gamma_{\mathrm{J}}$ and $\Gamma_{\mathrm{E}}$.

From (4.5), using successively, the definition of $\boldsymbol{E}$, a Green's formula, (3.20), (3.18), (3.23), (3.24) and Lemma 3.1, we have

$$
\begin{aligned}
-\sum_{n=1}^{N} V_{n}(t)\langle\operatorname{curl} \boldsymbol{G} \cdot \boldsymbol{n}, 1\rangle_{\Gamma_{\mathrm{J}}^{n}} & =\int_{\Omega} \mu \partial_{t} \boldsymbol{H}(t) \cdot \boldsymbol{G}+\int_{\Omega_{\mathrm{C}}} \frac{1}{\sigma} \operatorname{curl} \boldsymbol{H}(t) \cdot \operatorname{curl} \boldsymbol{G} \\
& =\int_{\Omega} \mu \partial_{t} \boldsymbol{H}(t) \cdot \boldsymbol{G}+\int_{\Omega} \boldsymbol{E}(t) \cdot \operatorname{curl} \boldsymbol{G} \\
& =\langle\boldsymbol{G} \times \boldsymbol{n}, \boldsymbol{n} \times \boldsymbol{E}(t) \times \boldsymbol{n}\rangle=-\left\langle\boldsymbol{G} \times \boldsymbol{n}, \operatorname{grad}_{\tau} \widetilde{V}(t)\right\rangle \\
& =-\sum_{n=1}^{N}\left(\left.\widetilde{V}(t)\right|_{\Gamma_{\mathrm{E}}^{n}}-\left.\widetilde{V}(t)\right|_{\Gamma_{\mathrm{J}}^{n}}\right)\langle\operatorname{curl} \boldsymbol{G} \cdot \boldsymbol{n}, 1\rangle_{\Gamma_{\mathrm{J}}^{n}} \quad \forall \boldsymbol{G} \in \mathcal{X} .
\end{aligned}
$$

Next, we take as test function $\boldsymbol{G}^{m} \in \mathcal{X}$ satisfying $\left\langle\operatorname{curl} \boldsymbol{G}^{m} \cdot \boldsymbol{n}, 1\right\rangle_{\Gamma^{n}}=\delta_{m n}, m, n=1, \ldots, N$ (see [9], Rem. 5.3 for the existence of such $\left.\boldsymbol{G}^{m}\right)$. By so doing, we obtain $L_{n}(\boldsymbol{E}(t))=\left.\widetilde{V}(t)\right|_{\Gamma_{\mathrm{J}}^{n}}-\left.\widetilde{V}(t)\right|_{\Gamma_{\mathrm{E}}^{n}}=V_{n}(t), n=1, \ldots, N$, from which it follows (4.1).

Finally, the proof of uniqueness of solution is identical to that in Theorem 3.4.

Remark 4.3. As in Section 3, we conclude that we can use the simplest choice of data $g=0$ on $\Gamma_{\mathrm{D}}$ without affecting the quantities of main interest, namely, $\boldsymbol{H}$ in the whole domain $\Omega$ and $\boldsymbol{E}$ in the conducting domain $\Omega_{\mathrm{C}}$.

Next step is the space discretization of Problem 4.1. Let $\mathcal{U}_{h}$ and $\mathcal{Q}_{h}$ be as in Subsection 3.1. Let $\mathcal{U}_{h}^{0}:=$ $\left\{\boldsymbol{w}_{h} \in \mathcal{U}_{h}: \widetilde{L}_{n}\left(\boldsymbol{w}_{h}\right)=0, n=1, \ldots, N\right\}$ with $\widetilde{L}_{n}$ as defined in (3.34). The space-discretization reads as follows:

Problem 4.4. Given $g \in \mathrm{L}^{2}\left(0, T ; L^{2}\left(\Gamma_{\mathrm{D}}\right)\right), V_{n} \in \mathrm{H}^{1}(0, T), n=1, \ldots, N$, and $\boldsymbol{H}_{0}$ satisfying (3.1), find $\boldsymbol{u}_{h}$ : $[0, T] \rightarrow \mathcal{U}_{h}$ and $\xi_{h}:[0, T] \rightarrow \mathcal{Q}_{h}$ such that

$$
\begin{aligned}
& \widetilde{L}_{n}\left(\boldsymbol{u}_{h}(t)\right)=\int_{0}^{t} V_{n}(s) \mathrm{d} s, \quad n=1, \ldots, N \\
& \int_{\Omega_{\mathrm{C}}} \sigma \partial_{t} \boldsymbol{u}_{h}(t) \cdot \boldsymbol{w}_{h}+\int_{\Omega} \frac{1}{\mu} \operatorname{curl} \boldsymbol{u}_{h}(t) \cdot \operatorname{curl} \boldsymbol{w}_{h}+\int_{\Omega_{\mathrm{D}}} \epsilon \boldsymbol{w}_{h} \cdot \operatorname{grad} \xi_{h}(t)=\int_{\Omega} \operatorname{curl} \boldsymbol{H}_{0} \cdot \boldsymbol{w}_{h} \quad \forall \boldsymbol{w}_{h} \in \mathcal{U}_{h}^{0}, \\
& \int_{\Omega_{\mathrm{D}}} \epsilon \boldsymbol{u}_{h}(t) \cdot \operatorname{grad} \varphi_{h}=\int_{\Gamma_{\mathrm{D}}}\left(\int_{0}^{t} g(s) \mathrm{d} s\right) \varphi_{h} \quad \forall \varphi_{h} \in \mathcal{Q}_{h}, \\
& \boldsymbol{u}_{h}(0)=\mathbf{0} \quad \text { in } \Omega .
\end{aligned}
$$


To prove that this problem is well-posed, our first step is to build an auxiliary function $\check{\boldsymbol{u}}_{h} \in \mathrm{H}^{1}\left(0, T ; \mathcal{U}_{h}\right)$ satisfying $\widetilde{L}_{n}\left(\check{\boldsymbol{u}}_{h}(t)\right)=\int_{0}^{t} V_{n}(s) \mathrm{d} s, n=1, \ldots, N, t \in[0, T]$. To define $\check{\boldsymbol{u}}_{h}$, first we choose functions $\boldsymbol{\Phi}_{m} \in \mathcal{U}_{h}$ such that $\widetilde{L}_{n}\left(\boldsymbol{\Phi}_{m}\right)=\delta_{m n}, m, n=1, \ldots, N$; such $\boldsymbol{\Phi}_{m}$ are easy to construct once a basis of $\mathcal{U}_{h}$ is given (see Rem. 5.1 below). Then, we define

$$
\check{\boldsymbol{u}}_{h}(t):=\sum_{m=1}^{N} \int_{0}^{t} V_{m}(s) \mathrm{d} s \boldsymbol{\Phi}_{m} .
$$

Hence,

$$
\widetilde{L}_{n}\left(\check{\boldsymbol{u}}_{h}(t)\right)=\sum_{m=1}^{N} \int_{0}^{t} V_{m}(s) \mathrm{d} s \widetilde{L}_{n}\left(\boldsymbol{\Phi}_{m}\right)=\int_{0}^{t} V_{n}(s) \mathrm{d} s .
$$

Moreover, since $V_{n} \in \mathrm{H}^{1}(0, T), n=1, \cdots, N$, we conclude that $\check{\boldsymbol{u}}_{h} \in \mathrm{H}^{1}\left(0, T ; \mathcal{U}_{h}\right)$.

Now, if we write $\boldsymbol{u}_{h}=\overline{\boldsymbol{u}}_{h}+\check{\boldsymbol{u}}_{h}$, Problem 4.4 is equivalent to finding $\overline{\boldsymbol{u}}_{h}:[0, T] \rightarrow \boldsymbol{U}_{h}^{0}$ and $\xi_{h}:[0, T] \rightarrow \mathcal{Q}_{h}$ such that

$$
\begin{aligned}
& \int_{\Omega_{\mathrm{C}}} \sigma \partial_{t} \overline{\boldsymbol{u}}_{h}(t) \cdot \boldsymbol{w}_{h}+\int_{\Omega} \frac{1}{\mu} \operatorname{curl} \overline{\boldsymbol{u}}_{h}(t) \cdot \operatorname{curl} \boldsymbol{w}_{h}+\int_{\Omega_{\mathrm{D}}} \epsilon \boldsymbol{w}_{h} \cdot \operatorname{grad} \xi_{h}(t) \\
& =\int_{\Omega_{\mathrm{C}}} \sigma \partial_{t} \check{\boldsymbol{u}}_{h}(t) \cdot \boldsymbol{w}_{h}-\int_{\Omega} \frac{1}{\mu} \operatorname{curl} \check{\boldsymbol{u}}_{h}(t) \cdot \operatorname{curl} \boldsymbol{w}_{h}+\int_{\Omega} \operatorname{curl} \boldsymbol{H}_{0} \cdot \boldsymbol{w}_{h} \quad \forall \boldsymbol{w}_{h} \in \mathcal{U}_{h}^{0}, \\
& \int_{\Omega_{\mathrm{D}}} \epsilon \overline{\boldsymbol{u}}_{h}(t) \cdot \operatorname{grad} \varphi_{h}=\int_{\Gamma_{\mathrm{D}}}\left(\int_{0}^{t} g(s) \mathrm{d} s\right) \varphi_{h}-\int_{\Omega_{\mathrm{D}}} \epsilon \check{\boldsymbol{u}}_{h}(t) \cdot \operatorname{grad} \varphi_{h} \quad \forall \varphi_{h} \in \mathcal{Q}_{h}, \\
& \overline{\boldsymbol{u}}_{h}(0)=\mathbf{0} \quad \text { in } \Omega .
\end{aligned}
$$

The well-posedness of this problem is obtained by following the same arguments used for Problem 3.6 in Section 3.1. The main difference is that now we need a discrete inf-sup condition similar to (3.38), but taking supremum in $\mathcal{U}_{h}^{0}$ instead of $\mathcal{U}_{h}$. However, for the proof of (3.38) it was used a function $\boldsymbol{w}_{h}=\operatorname{grad} \widetilde{\varphi}_{h}$ which actually lies in $\mathcal{U}_{h}^{0}$. Altogether, we conclude that Problem 4.4 has a unique solution.

Next, the arguments in Section 3.1 can be readily adapted to obtain the error estimate. With this aim, we need the following result.

Lemma 4.5. If $\boldsymbol{w} \in \mathrm{H}^{r}(\operatorname{curl} ; \Omega) \cap \mathcal{U}$ then $\widetilde{L}_{n}\left(\mathcal{I}_{h}^{\mathcal{N}} \boldsymbol{w}\right)=L_{n}(\boldsymbol{w}), n=1, \ldots, N$.

Proof. We recall that for $\boldsymbol{w} \in \mathcal{U}$ there exists a unique $W \in \mathcal{W}$ such that $\boldsymbol{n} \times \boldsymbol{w} \times \boldsymbol{n}=-\operatorname{grad}_{\tau} W$ on $\partial \Omega$ and $L_{n}(\boldsymbol{w})=\left.W\right|_{\Gamma_{\mathrm{E}}^{n}}-\left.W\right|_{\Gamma_{\mathrm{J}}^{n}}, n=1, \ldots, N\left(c f\right.$. (3.11)). On the other hand, for $\boldsymbol{w} \in \mathrm{H}^{r}(\mathbf{c u r l} ; \Omega)$ we have that $\left.\boldsymbol{w}\right|_{\partial \Omega} \in \mathrm{H}^{r-1 / 2}(\partial \Omega)^{3}$ and, hence, $W \in \mathrm{H}^{r+1 / 2}(\partial \Omega)$. Thus, $\boldsymbol{w}$ and $W$ are smooth enough to write

$$
\boldsymbol{n} \times \mathcal{I}_{h}^{\mathcal{N}} \boldsymbol{w} \times \boldsymbol{n}=\mathcal{I}_{h}^{\mathcal{N}_{2 D}}(\boldsymbol{n} \times \boldsymbol{w} \times \boldsymbol{n})=-\mathcal{I}_{h}^{\mathcal{N}}{ }^{2 D}\left(\operatorname{grad}_{\tau} W\right)=-\operatorname{grad}_{\tau}\left(\mathcal{I}_{h}^{\mathcal{L}} W\right) \quad \text { on } \partial \Omega,
$$

where $\mathcal{I}_{h}^{\mathcal{N}_{2 D}}$ and $\mathcal{I}_{h}^{\mathcal{L}}$ denote the two-dimensional Nédélec and Lagrange interpolant operators, respectively. Then,

$$
\widetilde{L}_{n}\left(\mathcal{I}_{h}^{\mathcal{N}} \boldsymbol{w}\right)=\int_{C_{n}} \mathcal{I}_{h}^{\mathcal{N}} \boldsymbol{w} \cdot \boldsymbol{t}=-\int_{C_{n}} \operatorname{grad}_{\tau}\left(\mathcal{I}_{h}^{\mathcal{L}} W\right) \cdot \boldsymbol{t}=\left.\mathcal{I}_{h}^{\mathcal{L}} W\right|_{\Gamma_{\mathrm{E}}^{n}}-\left.\mathcal{I}_{h}^{\mathcal{L}} W\right|_{\Gamma_{\mathrm{J}}^{n}}=\left.W\right|_{\Gamma_{\mathrm{E}}^{n}}-\left.W\right|_{\Gamma_{\mathrm{J}}^{n}}=L_{n}(\boldsymbol{w}) .
$$

Thus we conclude the proof.

Now we are in a position to prove the following error estimate. 
Theorem 4.6. Let $\boldsymbol{u}$ be the solution to Problem 4.1 and $\boldsymbol{u}_{h}$ that to Problem 4.4. If $\boldsymbol{u} \in \mathrm{H}^{1}\left(0, T ; \mathrm{H}^{r}(\mathbf{c u r l} ; \Omega)\right)$ with $r \in\left(\frac{1}{2}, 1\right]$, then there exists a constant $C>0$, independent of $h$, such that

$$
\begin{aligned}
& \sup _{0 \leq t \leq T}\left\|\boldsymbol{u}(t)-\boldsymbol{u}_{h}(t)\right\|_{\mathrm{L}^{2}\left(\Omega_{\mathrm{C}}\right)^{3}}^{2}+\sup _{0 \leq t \leq T}\left\|\operatorname{curl} \boldsymbol{u}(t)-\operatorname{curl} \boldsymbol{u}_{h}(t)\right\|_{\mathrm{L}^{2}(\Omega)^{3}}^{2}+\int_{0}^{T}\left\|\partial_{t}\left(\boldsymbol{u}(t)-\boldsymbol{u}_{h}(t)\right)\right\|_{\mathrm{L}^{2}\left(\Omega_{\mathrm{C}}\right)^{3}}^{2} \mathrm{~d} t \\
& \leq C h^{2 r}\left\{\sup _{0 \leq t \leq T}\|\boldsymbol{u}(t)\|_{\mathrm{H}^{r}(\mathbf{c u r l} ; \Omega)}^{2}+\int_{0}^{T}\left\|\partial_{t} \boldsymbol{u}(t)\right\|_{\mathrm{H}^{r}(\mathbf{c u r l} ; \Omega)}^{2} \mathrm{~d} t\right\} \\
& \leq C h^{2 r}\|\boldsymbol{u}\|_{\mathrm{H}^{1}\left(0, T ; \mathrm{H}^{r}(\mathbf{c u r l} ; \Omega)\right)}^{2} .
\end{aligned}
$$

Proof. As a first step, we need to prove the analogue to Lemma 3.12 for $\boldsymbol{u}$ and $\boldsymbol{u}_{h}$ being solution to Problem 4.1 and 4.4, respectively. The only difference in this proof is that, now, the test functions $\boldsymbol{w}_{h}$ in (3.50) lie in $\mathcal{U}_{h}^{0}$ instead of $\mathcal{U}_{h}$. Therefore, we need to ensure that $\boldsymbol{\delta}_{h}(t):=\mathcal{I}_{h}^{\mathcal{N}}(\boldsymbol{u}(t))-\boldsymbol{u}_{h}(t)$ and $\partial_{t} \boldsymbol{\delta}_{h}(t)$ belong to $\mathcal{U}_{h}^{0}$; namely, $\widetilde{L}_{n}\left(\boldsymbol{\delta}_{h}(t)\right)=\widetilde{L}_{n}\left(\partial_{t} \boldsymbol{\delta}_{h}(t)\right)=0$. The former follows from Lemma 4.5 and the fact that $L_{n}(\boldsymbol{u}(t))=\widetilde{L}_{n}\left(\boldsymbol{u}_{h}(t)\right)$ ( $c f$. the first equations in Problem 4.1 and 4.4). For the latter we use the same arguments and the assumption $\boldsymbol{u} \in \mathrm{H}^{1}\left(0, T ; \mathrm{H}^{r}(\mathbf{c u r l} ; \Omega)\right)$. The rest of the proof follows identically as that of Theorem 3.13.

Finally, we introduce the fully discrete approximation of Problem 4.1 defined as follows:

Problem 4.7. Given $V_{n} \in \mathrm{H}^{1}(0, T), n=1, \ldots, N$, and $\boldsymbol{H}_{0}$ satisfying (3.1), find $\boldsymbol{u}_{h}^{m} \in \mathcal{U}_{h}$ and $\xi_{h}^{m} \in \mathcal{Q}_{h}$, $m=1, \ldots, M$, such that

$$
\begin{aligned}
& \widetilde{L}_{n}\left(\boldsymbol{u}_{h}\left(t_{m}\right)\right)=\int_{0}^{t_{m}} V_{n}(s) \mathrm{d} s, \quad n=1, \ldots, N, \\
& \int_{\Omega_{\mathrm{C}}} \sigma \frac{\boldsymbol{u}_{h}^{m}-\boldsymbol{u}_{h}^{m-1}}{\Delta t} \cdot \boldsymbol{w}_{h}+\int_{\Omega} \frac{1}{\mu} \operatorname{curl} \boldsymbol{u}_{h}^{m} \cdot \operatorname{curl} \boldsymbol{w}_{h}+\int_{\Omega_{\mathrm{D}}} \epsilon \boldsymbol{w}_{h} \cdot \operatorname{grad} \xi_{h}^{m}=\int_{\Omega} \operatorname{curl} \boldsymbol{H}_{0} \cdot \boldsymbol{w}_{h} \quad \forall \boldsymbol{w}_{h} \in \mathcal{U}_{h}^{0}, \\
& \int_{\Omega_{\mathrm{D}}} \epsilon \boldsymbol{u}_{h}^{m} \cdot \operatorname{grad} \varphi_{h}=\int_{\Gamma_{\mathrm{D}}}\left(\int_{0}^{t_{m}} g(s) \mathrm{d} s\right) \varphi_{h} \quad \forall \varphi_{h} \in \mathcal{Q}_{h}, \\
& \boldsymbol{u}_{h}^{0}=\mathbf{0} \quad \text { in } \Omega .
\end{aligned}
$$

This problem has a unique solution. In fact, taking $\check{\boldsymbol{u}}_{h}^{m}:=\check{\boldsymbol{u}}_{h}\left(t^{m}\right)$, with $\check{\boldsymbol{u}}_{h}$ as in (4.7), and writing $\boldsymbol{u}_{h}^{m}=$ $\overline{\boldsymbol{u}}_{h}^{m}+\check{\boldsymbol{u}}_{h}^{m}$, the $m$-th step of Problem 4.7 is equivalent to find $\overline{\boldsymbol{u}}_{h}^{m} \in \mathcal{U}_{h}^{0}$ and $\xi_{h}^{m} \in \mathcal{Q}_{h}$ such that

$$
\begin{aligned}
& \int_{\Omega_{\mathrm{C}}} \sigma \overline{\boldsymbol{u}}_{h}^{m} \cdot \boldsymbol{w}_{h}+\Delta t \int_{\Omega} \frac{1}{\mu} \operatorname{curl} \overline{\boldsymbol{u}}_{h}^{m} \cdot \operatorname{curl} \boldsymbol{w}_{h}+\Delta t \int_{\Omega_{\mathrm{D}}} \epsilon \boldsymbol{w}_{h} \cdot \operatorname{grad} \xi_{h}^{m} \\
& =\int_{\Omega_{\mathrm{C}}} \sigma \overline{\boldsymbol{u}}_{h}^{m-1} \cdot \boldsymbol{w}_{h}+\Delta t \int_{\Omega} \operatorname{curl} \boldsymbol{H}_{0} \cdot \boldsymbol{w}_{h}-\int_{\Omega_{\mathrm{C}}} \sigma\left(\check{\boldsymbol{u}}_{h}^{m}-\check{\boldsymbol{u}}_{h}^{m-1}\right) \cdot \boldsymbol{w}_{h}-\Delta t \int_{\Omega} \frac{1}{\mu} \operatorname{curl} \check{\boldsymbol{u}}_{h}^{m} \cdot \operatorname{curl} \boldsymbol{w}_{h} \quad \forall \boldsymbol{w}_{h} \in \mathcal{U}_{h}^{0}, \\
& \Delta t \int_{\Omega_{\mathrm{D}}} \epsilon \overline{\boldsymbol{u}}_{h}^{m} \cdot \operatorname{grad} \varphi_{h}=\Delta t \int_{\Gamma_{\mathrm{D}}}\left(\int_{0}^{t_{m}} g(s) \mathrm{d} s\right) \varphi_{h}+\Delta t \int_{\Omega_{\mathrm{D}}} \epsilon \check{\boldsymbol{u}}_{h}^{m} \cdot \operatorname{grad} \varphi_{h} \quad \forall \varphi_{h} \in \mathcal{Q}_{h} .
\end{aligned}
$$

The well-posedness of this problem follows identically as that of Problem 3.16. The same happens with the error estimates analogous to those in Theorem 3.18. Therefore, we conclude the following result.

Theorem 4.8. Let $\boldsymbol{u}$ be the solution to Problem 4.1 and $\boldsymbol{u}_{h}^{k}, k=1, \ldots, M$, that to Problem 4.7. If $\boldsymbol{u} \in$ $\mathrm{H}^{1}\left(0, T ; \mathrm{H}^{r}(\operatorname{curl} ; \Omega)\right)$ for $r \in\left(\frac{1}{2}, 1\right]$ and $\left.\boldsymbol{u}\right|_{\Omega_{\mathrm{C}}} \in \mathrm{H}^{2}\left(0, T ; \mathrm{L}^{2}\left(\Omega_{\mathrm{C}}\right)^{3}\right)$, then there exists a constant $C>0$, 
independent of $h$ and $\Delta t$, such that

$$
\begin{aligned}
& \max _{1 \leq k \leq M}\left\|\boldsymbol{u}\left(t_{k}\right)-\boldsymbol{u}_{h}^{k}\right\|_{\mathrm{L}^{2}\left(\Omega_{\mathrm{C}}\right)^{3}}^{2}+\max _{1 \leq k \leq M}\left\|\operatorname{curl}\left(\boldsymbol{u}\left(t_{k}\right)-\boldsymbol{u}_{h}^{k}\right)\right\|_{\mathrm{L}^{2}(\Omega)^{3}}^{2}+\Delta t \sum_{k=1}^{M}\left\|\partial_{t} \boldsymbol{u}\left(t_{k}\right)-\frac{\boldsymbol{u}_{h}^{k}-\boldsymbol{u}_{h}^{k-1}}{\Delta t}\right\|_{\mathrm{L}^{2}\left(\Omega_{\mathrm{C}}\right)^{3}}^{2} \\
& \quad \leq C\left\{(\Delta t)^{2} \int_{0}^{T}\left\|\partial_{t t} \boldsymbol{u}(t)\right\|_{\mathrm{L}^{2}\left(\Omega_{\mathrm{C}}\right)^{3}}^{2} \mathrm{~d} t+h^{2 r} \sup _{0 \leq t \leq T}\|\boldsymbol{u}(t)\|_{\mathrm{H}^{r}(\mathbf{c u r l} ; \Omega)}^{2}+h^{2 r} \int_{0}^{T}\left\|\partial_{t} \boldsymbol{u}(t)\right\|_{\mathrm{H}^{r}(\mathbf{c u r l} ; \Omega)}^{2} \mathrm{~d} t\right\} \\
& \quad \leq C\left\{(\Delta t)^{2}\|\boldsymbol{u}\|_{\mathrm{H}^{2}\left(0, T ; \mathrm{L}^{2}\left(\Omega_{\mathrm{C}}\right)^{3}\right)}^{2}+h^{2 r}\|\boldsymbol{u}\|_{\mathrm{H}^{1}\left(0, T ; \mathrm{H}^{r}(\mathbf{c u r l} ; \Omega)\right)}^{2}\right\} .
\end{aligned}
$$

Remark 4.9. As in the case of Problem 3.16, we approximate the electric field $\boldsymbol{E}$ and the magnetic field $\boldsymbol{H}$ at each time $t_{k}, k=1, \ldots, M$, by means of $\boldsymbol{E}_{h}^{k}:=\frac{\boldsymbol{u}_{h}^{k}-\boldsymbol{u}_{h}^{k-1}}{\Delta t}$ and $\boldsymbol{H}_{h}^{k}:=\frac{1}{\mu} \operatorname{curl} \boldsymbol{u}_{h}^{k}-\boldsymbol{H}_{0}$, respectively. Then, Theorem 4.8 yields the following error estimates:

$$
\begin{aligned}
& \Delta t \sum_{k=1}^{M}\left\|\boldsymbol{E}\left(t_{k}\right)-\boldsymbol{E}_{h}^{k}\right\|_{\mathrm{L}^{2}\left(\Omega_{\mathrm{C}}\right)^{3}}^{2} \leq C\left\{(\Delta t)^{2}\|\boldsymbol{u}\|_{\mathrm{H}^{2}\left(0, T ; \mathrm{L}^{2}\left(\Omega_{\mathrm{C}}\right)^{3}\right)}^{2}+h^{2 r}\|\boldsymbol{u}\|_{\mathrm{H}^{1}\left(0, T ; \mathrm{H}^{r}(\operatorname{curl} ; \Omega)\right)}^{2}\right\}, \\
& \left.\max _{1 \leq k \leq M} \| \boldsymbol{H}\left(t_{k}\right)-\boldsymbol{H}_{h}^{k}\right) \|_{\mathrm{L}^{2}(\Omega)^{3}}^{2} \leq C\left\{(\Delta t)^{2}\|\boldsymbol{u}\|_{\mathrm{H}^{2}\left(0, T ; \mathrm{L}^{2}\left(\Omega_{\mathrm{C}}\right)^{3}\right)}^{2}+h^{2 r}\|\boldsymbol{u}\|_{\mathrm{H}^{1}\left(0, T ; \mathrm{H}^{r}(\operatorname{curl} ; \Omega)\right)}^{2}\right\} .
\end{aligned}
$$

Remark 4.10. The same observation made in Remark 3.15 holds in this case.

Let us remark that the constraints $\widetilde{L}_{n}\left(\boldsymbol{u}_{h}\left(t_{m}\right)\right)=\int_{0}^{t_{m}} V_{n}(s) d s, n=1, \ldots, N$, can be imposed by means of a Lagrange multiplier. In such a case, we are led to the following problem:

Problem 4.11. Given $V_{n} \in \mathrm{H}^{1}(0, T), n=1, \ldots, N$, and $\boldsymbol{H}_{0}$ satisfying (3.1), find $\boldsymbol{u}_{h}^{m} \in \mathcal{U}_{h}, \xi_{h}^{m} \in \mathcal{Q}_{h}$ and $\mathbb{I}^{m}=\left(\mathbb{I}_{1}^{m}, \ldots, \mathbb{I}_{N}^{m}\right) \in \mathbb{R}^{N}, m=1, \ldots, M$, such that

$$
\begin{aligned}
& \int_{\Omega_{\mathrm{C}}} \sigma \frac{\boldsymbol{u}_{h}^{m}-\boldsymbol{u}_{h}^{m-1}}{\Delta t} \cdot \boldsymbol{w}_{h}+\int_{\Omega} \frac{1}{\mu} \operatorname{curl} \boldsymbol{u}_{h}^{m} \cdot \operatorname{curl} \boldsymbol{w}_{h}+\int_{\Omega_{\mathrm{D}}} \epsilon \boldsymbol{w}_{h} \cdot \operatorname{grad} \xi_{h}^{m}+\sum_{n=1}^{N} \mathbb{I}_{n}^{m} \widetilde{L}_{n}\left(\boldsymbol{w}_{h}\right) \\
& =\int_{\Omega} \operatorname{curl} \boldsymbol{H}_{0} \cdot \boldsymbol{w}_{h} \quad \forall \boldsymbol{w}_{h} \in \mathcal{U}_{h}, \\
& \int_{\Omega_{\mathrm{D}}} \epsilon \boldsymbol{u}_{h}^{m} \cdot \operatorname{grad} \varphi_{h}=\int_{\Gamma_{\mathrm{D}}}\left(\int_{0}^{t_{m}} g(s) \mathrm{d} s\right) \varphi \quad \forall \varphi_{h} \in \mathcal{Q}_{h}, \\
& \sum_{n=1}^{N} \widetilde{L}_{n}\left(\boldsymbol{u}_{h}^{m}\right) \mathbb{J}_{n}=\sum_{n=1}^{N} \int_{0}^{t} V_{n}(s) \mathrm{d} s \mathbb{J}_{n} \quad \forall \mathbb{J}=\left(\mathbb{J}_{1}, \ldots, \mathbb{J}_{N}\right) \in \mathbb{R}^{N}, \\
& \boldsymbol{u}_{h}^{0}=\mathbf{0} \quad \text { in } \Omega \text {. }
\end{aligned}
$$

The following lemma shows that this and Problem 4.7 are actually equivalent:

Lemma 4.12. Given $V_{n} \in \mathrm{H}^{1}(0, T), n=1, \ldots, N$, and $\boldsymbol{H}_{0}$ satisfying $(3.1),\left(\boldsymbol{u}_{h}^{m}, \xi_{h}^{m}\right), m=1, \ldots, M$, is the solution to Problem 4.7 if and only if there exist $\mathbb{I}^{m} \in \mathbb{R}^{N}$ such that $\left(\boldsymbol{u}_{h}^{m}, \xi_{h}^{m}, \mathbb{I}^{m}\right), m=1, \ldots, M$, is the unique solution to Problem 4.11.

Proof. The result is a consequence of the existence and uniqueness of the solution to Problem 4.7 and the fact that the bilinear form $c: \mathcal{U}_{h} \times \mathbb{R}^{N} \rightarrow \mathbb{R}$ defined by $c\left(\boldsymbol{w}_{h}, \mathbb{J}\right):=\sum_{n=1}^{N} \widetilde{L}_{n}\left(\boldsymbol{w}_{h}\right) \mathbb{J}_{n}$ satisfies a discrete inf-sup condition, see [11], Lemma 3.3 .

The Lagrange multipliers $\mathbb{I}_{n}^{m}$ have a physical meaning. In fact, the equations of Problem 4.11 are exactly the same as those of Problem 3.16, with $\mathbb{I}_{n}^{m}$ instead of $I_{n}(0)-I_{n}\left(t_{m}\right)$. Therefore by solving Problem 4.11, we can compute the input currents on each conductor $\Omega_{\mathrm{C}}^{n}$ by means of $I_{n}\left(t_{m}\right)=I_{n}(0)-\mathbb{I}_{n}^{m}$ (provided $I_{n}(0)$ is known). 


\section{NumERICAL EXPERIMENTS}

In this section we present some numerical results obtained with a MATLAB code implementing the numerical method described above. First, we give some details about the computer implementation. Then, we present a test with a known analytical solution which we use to validate the computer code and to check the error estimates proved above. Finally, we apply the method to a problem in a more realistic geometry.

\subsection{Implementation issues}

We have implemented in our codes matrix forms of Problem 3.6 and 4.11. In both cases we need a basis of $\mathcal{U}_{h}$. We have used the following one taken from [11], Section 3.

$$
\left\{\Phi_{e}: e \in \stackrel{\circ}{\mathcal{E}}_{h}\right\} \cup\left\{\operatorname{grad} \varphi_{v}: v \in \mathcal{V}_{h}^{\Gamma_{\mathrm{D}}}\right\} \cup\left\{\operatorname{grad} \varphi_{n}^{\mathrm{J}}: n=1, \ldots, N\right\} \cup\left\{\operatorname{grad} \varphi_{n}^{\mathrm{E}}: n=1, \ldots, N\right\},
$$

where

- $\stackrel{\circ}{\mathcal{E}}_{h}$ is the set of inner edges of the mesh $\mathcal{T}_{h}$ (i.e., edges $\left.e \nsubseteq \partial \Omega\right)$ and, for each $e \in \stackrel{\circ}{\mathcal{E}}_{h}, \boldsymbol{\Phi}_{e} \in \mathcal{N}_{h}(\Omega)$ is the Nedéléc basis function associated to $e$;

- $\mathcal{V}_{h}^{\Gamma_{\mathrm{D}}}$ is the set of vertices of the mesh $\mathcal{T}_{h}$ lying on the open surface $\Gamma_{\mathrm{D}}$ and, for all vertices $v \in \bar{\Omega}_{\mathrm{D}}, \varphi_{v} \in \mathcal{L}_{h}\left(\Omega_{\mathrm{D}}\right)$ is the piecewise linear function associated to $v$;

- $\varphi_{n}^{\mathrm{J}}$ is the piecewise linear function such that $\varphi_{n}^{\mathrm{J}}=1$ for all vertices of the mesh $\mathcal{T}_{h}$ lying on the closed surface $\bar{\Gamma}_{\mathrm{J}}^{n}$ and $\varphi_{n}^{\mathrm{J}}=0$ otherwise;

- $\varphi_{n}^{\mathrm{E}}$ is the piecewise linear function such that $\varphi_{n}^{\mathrm{E}}=1$ for all vertices of the mesh $\mathcal{T}_{h}$ lying on the closed surface $\bar{\Gamma}_{\mathrm{E}}^{n}$ and $\varphi_{n}^{\mathrm{E}}=0$ otherwise.

In spite of the fact that $\widetilde{L}_{n}$ is defined by means of an integral on a particular curve $C_{n}(c f .(3.34))$, in practice, there is no need to construct such curves. In fact, to impose the constraint $\widetilde{L}_{n}\left(\boldsymbol{u}_{h}\left(t_{k}\right)\right)=\int_{0}^{t_{k}} V_{n}(s) \mathrm{d} s$, it is enough to evaluate $\widetilde{L}_{n}$ for the basis functions of $\mathcal{U}_{h}$ by means of (3.34). Thus, we obtain

$$
\widetilde{L}_{n}\left(\boldsymbol{w}_{h}\right)=\left\{\begin{array}{cl}
0, & \text { if } \boldsymbol{w}_{h}=\boldsymbol{\Phi}_{e}, \\
0, & \text { if } \boldsymbol{w}_{h}=\operatorname{grad} \varphi_{v} \text { for } v \in \mathcal{V}_{h}^{\Gamma_{\mathrm{D}}}, \\
\delta_{m n}, & \text { if } \boldsymbol{w}_{h}=\operatorname{grad} \varphi_{m}^{\mathrm{J}}, \\
-\delta_{m n}, & \text { if } \boldsymbol{w}_{h}=\operatorname{grad} \varphi_{m}^{\mathrm{E}} .
\end{array}\right.
$$

On the other hand, a basis of $\mathcal{Q}_{h}$ is given by

$$
\left\{\varphi_{v}: v \in \mathcal{V}_{h}^{\Gamma_{\mathrm{D}}}\right\} \cup\left\{\varphi_{v}: v \in \Omega_{\mathrm{D}}\right\} \cup\left\{\varphi_{k}: k=2, \ldots, M\right\}
$$

where $\varphi_{v}$ are as defined above and $\varphi_{k}$ is the piecewise linear function such that $\varphi_{k}=1$ for all vertices of the mesh $\mathcal{T}_{h}$ lying on the closed surface $\bar{\Gamma}_{\mathrm{I}}^{k}, k=2, \ldots, M$, and vanishing at all the other vertices.

Remark 5.1. Let us recall that to prove that Problem 4.4 is well-posed, we have used functions $\boldsymbol{\Phi}_{m}$ satisfying $\widetilde{L}_{n}\left(\boldsymbol{\Phi}_{m}\right)=\delta_{m n}, m, n=1, \ldots, N$. An example of one such $\boldsymbol{\Phi}_{m}$ is defined by $\boldsymbol{\Phi}_{m}:=\operatorname{grad} \varphi_{m}^{J}$, where $\varphi_{m}^{J}$ is as above.

\subsection{A test with known analytical solution}

To test our codes, we applied the proposed method to the same problem solved in [10] in harmonic regime. This is the reason why we only give here a brief description and refer the reader to the quoted paper for further details. Figure 2 shows a sketch of the domain where the conducting part $\Omega_{\mathrm{C}}$ and the whole domain $\Omega$ are coaxial cylinders of respective radius $R_{\mathrm{C}}=0.25 \mathrm{~m}$ and $R_{\mathrm{D}}=0.5 \mathrm{~m}$ and height $A=0.5 \mathrm{~m}$. First, we solve the problem with input intensity as boundary data. An alternating current of intensity $I(t)=I_{0} \cos (\omega t)$ enters the 

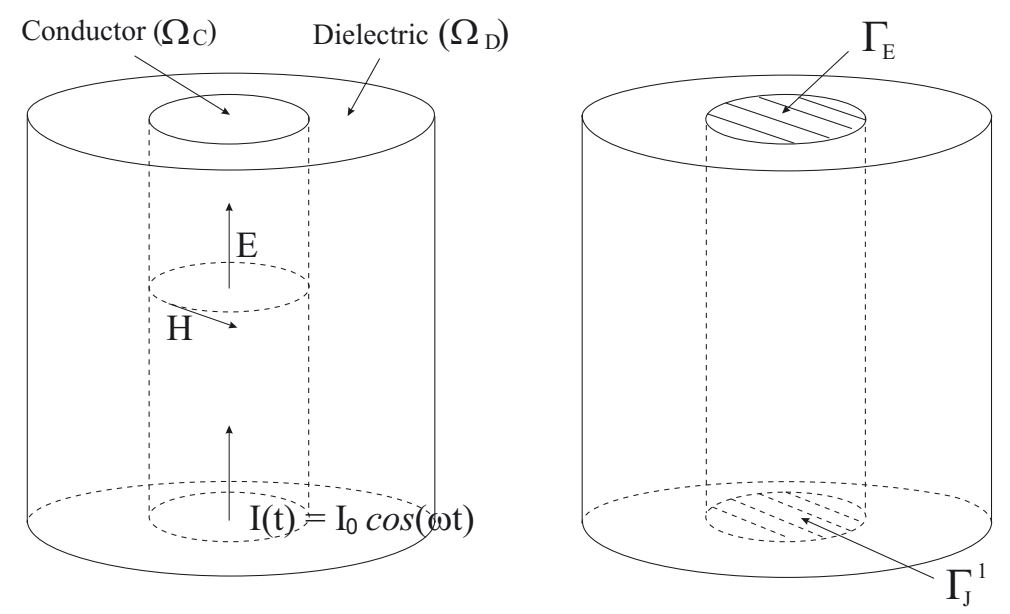

FiguRE 2. Sketch of the domain in the analytical example.

conductor through $\Gamma_{\mathrm{J}}^{1}$ and crosses $\Omega_{\mathrm{C}}$ in the axial direction; $I_{0}$ denotes the amplitude of the intensity and $\omega$ the angular frequency. Under these assumptions, by using a cylindrical coordinate system, it is easy to obtain an analytical solution of the eddy current problem in $\Omega$ by writing all the fields in the form $\boldsymbol{F}(t, \boldsymbol{x})=\operatorname{Re}\left(e^{\mathrm{i} \omega t} \mathcal{F}(\boldsymbol{x})\right)$.

To solve Problem 3.16 we also need the data $g \in \mathrm{L}^{2}\left(0, T ; \mathrm{L}^{2}\left(\Gamma_{\mathrm{D}}\right)\right)$. However, as stated above, the most relevant physical quantities $\boldsymbol{H}$ and $\left.\boldsymbol{E}\right|_{\Omega_{\mathrm{C}}}$ are independent of the chosen $g$. Because of this, we have solved Problem 3.16 by means of the easiest choice: $g=0$.

The numerical method has been used on several successively refined meshes and the time-step has been conveniently reduced to analyze the convergence with respect to both, the mesh-size and the time-step simultaneously. We have compared the obtained numerical solutions with the analytical one.

In order to show the linear convergence with respect to the mesh-size and the time-step, we have computed the relative errors of the different fields corresponding to $\frac{h}{n}, \frac{\Delta t}{n}, n=1, \ldots, 7$. Figure 3 shows $\log$ - $\log$ plots of the relative error for the physical variables of interest, the magnetic field and the electric field in the conductor domain, in the discrete norms considered in Remark 3.19 versus the number of degrees of freedom (d.o.f.).

Secondly we consider voltage drops as boundary data for the same problem. In this case it is easy to show that the corresponding voltage drop is given by $V(t)=\operatorname{Re}\left(e^{\mathrm{i} \omega t} \mathcal{V}\right)$ (see [5], Sect. 8.1.5), where

$$
\mathcal{V}=\frac{\gamma A I_{0}}{2 \pi \sigma R_{C}} \frac{\mathcal{I}_{0}\left(\gamma R_{C}\right)}{\mathcal{I}_{1}\left(\gamma R_{C}\right)}+\mathrm{i} \omega \mu \frac{A I_{0}}{2 \pi} \log \left(\frac{R_{D}}{R_{C}}\right)
$$

with $\gamma=\sqrt{i \omega \mu \sigma}$ and $\mathcal{I}_{0}, \mathcal{I}_{1}$ the Bessel's function of order 0 and 1, respectively.

We have compared the obtained numerical solutions with the analytical one. As in the previous case, we have chosen $g=0$. Figure 4 shows log-log plots of the relative errors for the magnetic field and for the electric field in $\Omega_{\mathrm{C}}$ in the discrete norms considered in Remark 4.9 versus the number of degrees of freedom.

In both tests, with intensities or voltage drops as boundary data, the error curves show a very good agreement with the theoretically predicted order of convergence. In fact, the relative error of $\boldsymbol{H}$ behaves always very close to $\mathcal{O}(h+\Delta t)$. The order of convergence of $\boldsymbol{E}$ is initially worse (although the relative errors are smaller than those of $\boldsymbol{H})$ but finally it is also almost $\mathcal{O}(h+\Delta t)$. Moreover, these results are actually independent of the choice of $g$. In fact, we have also solved Problems 3.6 and 4.4 with two other choices of $g$ : a random one and the exact value of $\left.\epsilon \boldsymbol{E}\right|_{\Gamma_{\mathrm{D}}}$ (which was obtained by analytical computations similar to those in [5], Sect. 8.1.5). In all cases the computed values of $\boldsymbol{H}_{h}^{k}$ and $\boldsymbol{E}_{h}^{k}$, the latter only in $\Omega_{\mathrm{C}}$, coincide up to rounding errors. 


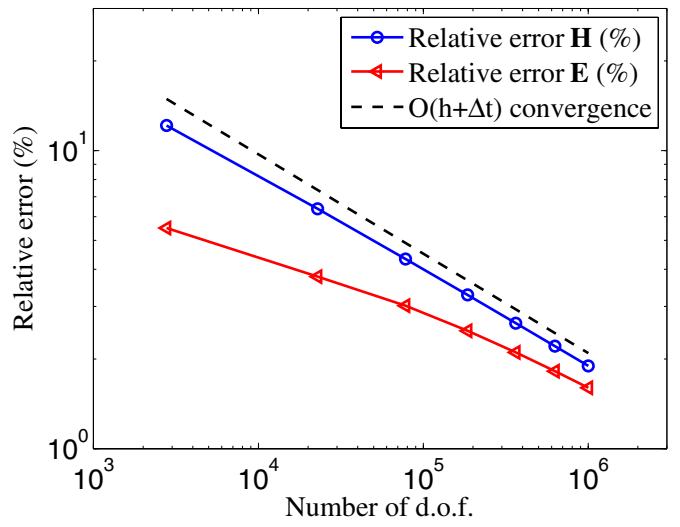

FIGURE 3. $\frac{\left.\max _{1 \leq k \leq M} \| \boldsymbol{H}\left(t_{k}\right)-\boldsymbol{H}_{h}^{k}\right) \|_{\mathrm{L}^{2}(\Omega)^{3}}}{\max _{1 \leq k \leq M}\left\|\boldsymbol{H}\left(t_{k}\right)\right\|_{\mathrm{L}^{2}(\Omega)^{3}}}$ and $\frac{\sqrt{\Delta t}\left\{\sum_{k=1}^{M}\left\|\boldsymbol{E}\left(t_{k}\right)-\boldsymbol{E}_{h}^{k}\right\|_{\mathrm{L}^{2}\left(\Omega_{\mathrm{C}}\right)^{3}}^{2}\right\}^{1 / 2}}{\sqrt{\Delta t}\left\{\sum_{k=1}^{M}\left\|\boldsymbol{E}\left(t_{k}\right)\right\|_{\mathrm{L}^{2}\left(\Omega_{\mathrm{C}}\right)^{3}}^{2}\right\}^{1 / 2}}$ versus number of d.o.f. (log-log scale).

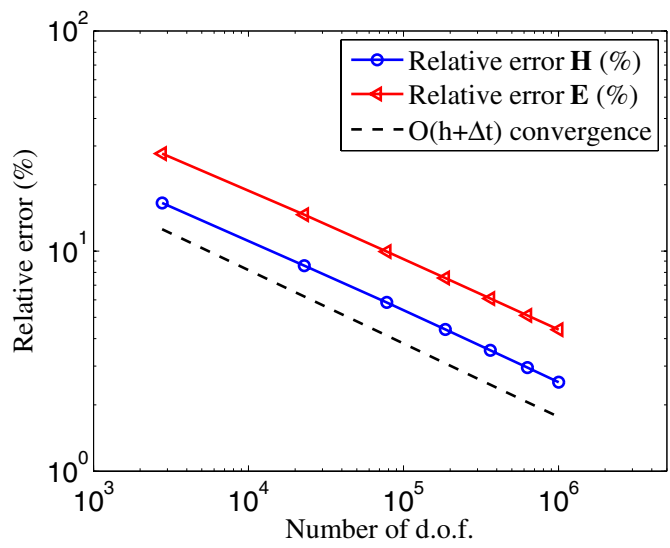

FIGURE 4. $\frac{\left.\max _{1 \leq k \leq M} \| \boldsymbol{H}\left(t_{k}\right)-\boldsymbol{H}_{h}^{k}\right) \|_{\mathrm{L}^{2}(\Omega)^{3}}}{\max _{1 \leq k \leq M}\left\|\boldsymbol{H}\left(t_{k}\right)\right\|_{\mathrm{L}^{2}(\Omega)^{3}}}$ and $\frac{\sqrt{\Delta t}\left\{\sum_{k=1}^{M}\left\|\boldsymbol{E}\left(t_{k}\right)-\boldsymbol{E}_{h}^{k}\right\|_{\mathrm{L}^{2}\left(\Omega_{\mathrm{C}}\right)^{3}}^{2}\right\}^{1 / 2}}{\sqrt{\Delta t}\left\{\sum_{k=1}^{M}\left\|\boldsymbol{E}\left(t_{k}\right)\right\|_{\mathrm{L}^{2}\left(\Omega_{\mathrm{C}}\right)^{3}}^{2}\right\}^{1 / 2}}$ versus number of d.o.f.

(log-log scale).

Additionally, when the exact value of $g=\left.\epsilon \boldsymbol{E}\right|_{\Gamma_{\mathrm{D}}}$ was used, we have tested whether the computed values $\frac{\boldsymbol{u}_{h}^{k}-\boldsymbol{u}_{h}^{k-1}}{\Delta t}$ approximate the exact electric fields $\boldsymbol{E}_{\mathrm{D}}$ in $\Omega_{\mathrm{D}}$. In this case, although the theoretical results only guarantees such a convergence in $\Omega_{\mathrm{C}}$, we checked an $\mathcal{O}(h+\Delta t)$ convergence, too.

\subsection{A problem in a more realistic geometry}

In this section we have computed the eddy currents induced by a coil in a metallic plate. The coil and the plate are shown in Figure 5, which also shows a typical mesh of the conducting domain. Such configuration 

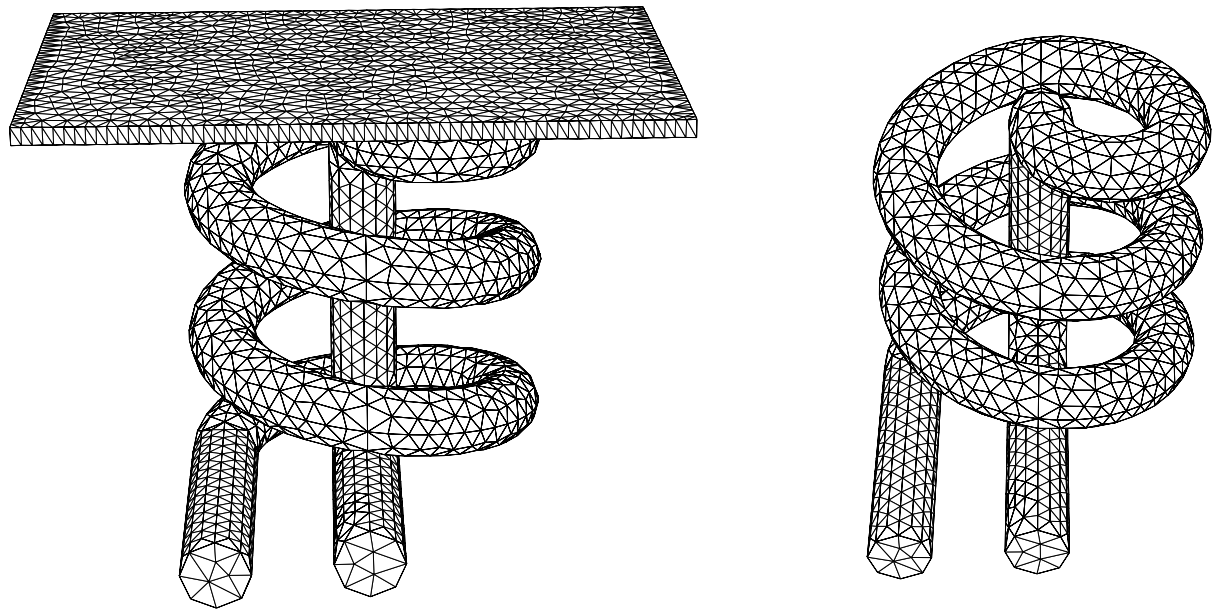

Figure 5. Mesh of the conducting domain (left). Detail of the coil mesh (right).

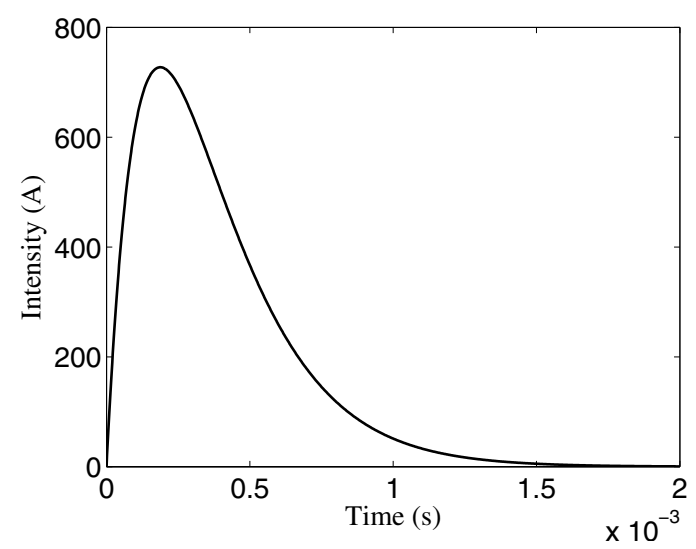

FiguRE 6. Imposed current intensity (A) vs. time (s).

is usually found, for instance, in problems related to non destructive testing or electromagnetic forming (see, e.g., $[25])$.

Domain $\Omega$ has been chosen as a sufficiently large box surrounding the conductor. Notice that in order to introduce a scalar potential in the dielectric domain to use the formulation proposed in [9], we would need to build a cutting surface in this domain, what would not be easy in this case.

The current intensity which enters the coil is shown in Figure 6 . Here, we have used $g=0$ on $[0, T] \times \Gamma_{\mathrm{D}}$, too.

In this test, the eddy currents induced in the plate are in the range $2.7 \times 10^{4}-1.8 \times 10^{3} \mathrm{~A} / \mathrm{m}^{2}$. They are significantly smaller than those in the coil (range $2.9 \times 10^{8}-8.1 \times 10^{8} \mathrm{~A} / \mathrm{m}^{2}$ ). This is the reason why we show coil and plate on separate figures. Figure 7 shows the modulus of the current density in the conducting domain. Figures 8 and 9 show the current density vector field. All the reported results correspond to the time at which the input current intensity reaches its maximum $(0.00018 \mathrm{~s})$. 

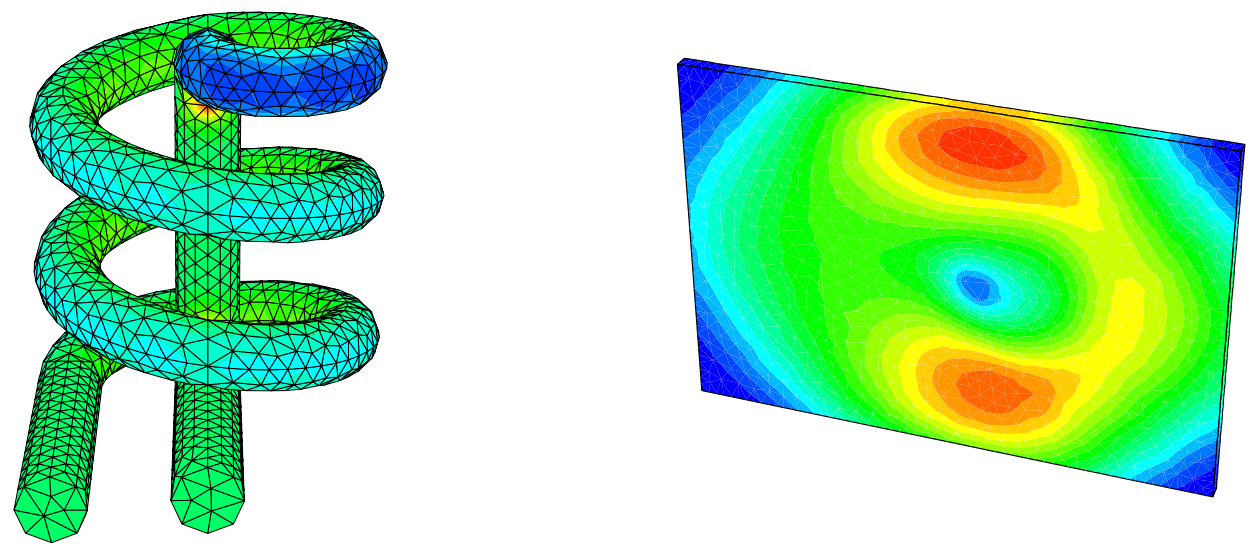

Figure 7. Modulus of the current density in coil and plate at time $0.00018 \mathrm{~s}$ (different scales).

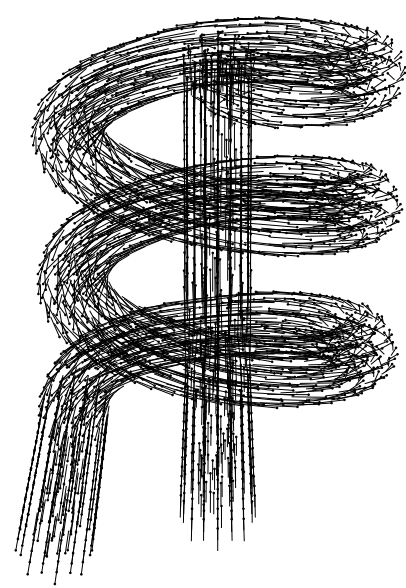

Figure 8. Distribution of the current density (vector field) in coil at time $0.00018 \mathrm{~s}$.

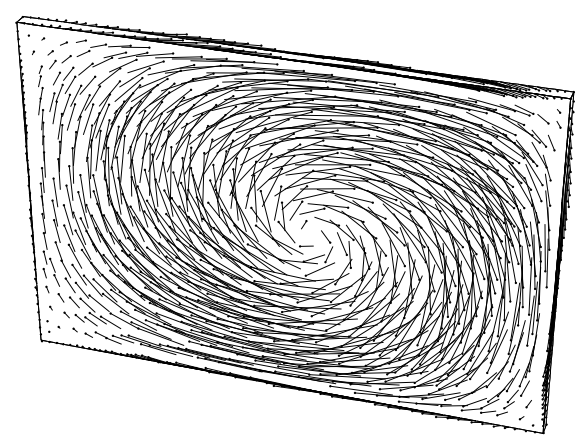

Figure 9. Distribution of the current density (vector field) in the plate at time $0.00018 \mathrm{~s}$. 


\section{REFERENCES}

[1] R. Acevedo, S. Meddahi and R. Rodríguez, An E-based mixed formulation for a time-dependent eddy current problem. Math. Comput. 78 (2009) 1929-1949.

[2] A. Alonso Rodríguez, R. Hiptmair and A. Valli, A hybrid formulation of eddy current problems. Numer. Methods Part. Differ. Equ. 21 (2005) 742-763.

[3] A. Alonso Rodríguez, R. Hiptmair and A. Valli, Mixed finite element approximation of eddy current problems. IMA J. Numer. Anal. 24 (2004) 255-271.

[4] A. Alonso and A. Valli, An optimal decomposition preconditioner for low-frequency time-harmonic Maxwell equations. Math. Comput. 68 (1999) 607-631.

[5] A. Alonso and A. Valli, Eddy Current Approximation of Maxwell Equations: Theory, Algorithms and Applications. SpringerVerlag, Italia (2010).

[6] A. Alonso Rodríguez and A. Valli, Voltage and current excitation for time-harmonic eddy-current problems. SIAM J. Appl. Math. 68 (2008) 1477-1494.

[7] C. Amrouche, C. Bernardi, M. Dauge and V. Girault, Vector potentials in three-dimensional non-smooth domains. Math. Methods Appl. Sci. 21 (1998) 823-864.

[8] A. Beranúdez, B. López-Rodríguez, R. Rodríguez and P. Salgado, Equivalence between two finite element methods for the eddy current problem. C. R. Math. Acad. Sci. Paris, Series I 34 (2010) 769-774.

[9] A. Bermúdez, B. López-Rodríguez, R. Rodríguez and P. Salgado, Numerical solution of transient eddy current problems with input current intensities as boundary data. IMA J. Numer. Anal. 32 (2012) 1001-1029.

[10] A. Bermúdez, R. Rodríguez and P. Salgado, A finite element method with Lagrange multipliers for low-frequency harmonic Maxwell equations. SIAM J. Numer. Anal. 40 (2002) 1823-1849.

[11] A. Bermúdez, R. Rodríguez and P. Salgado, Numerical analysis of electric field formulations of the eddy current model. Numer. Math. 102 (2005) 181-201.

[12] A. Bossavit, Computational Electromagnetism. Variational Formulations, Complementarity, Edge Elements. Academic Press, San Diego (1998).

[13] A. Bossavit, Most general non-local boundary conditions for the Maxwell equation in a bounded region. COMPEL 19 (2000) 239-245.

[14] A. Buffa, M. Costabel and D. Sheen, On traces for $\mathrm{H}(\mathbf{c u r l} ; \Omega)$ in Lipschitz domains. J. Math. Anal. Appl. 276 (2002) $845-876$.

[15] A. Buffa, Y. Maday and F. Rapetti, Applications of the mortar element method to 3D electromagnetic moving structures. Computational Electromagnetics, edited by C. Carstensen et al., Springer Verlag. Lect. Notes Comput. Sci. Eng. 28 (2003) $35-50$.

[16] C.R.I. Emson and J. Simkin, An optimal method for 3D eddy currents. IEEE Trans. Magn. 19 (1983) $2450-2452$.

[17] P. Fernandes and G. Gilardi, Magnetostatic and electrostatic problems in inhomogeneous anisotropic media with irregular boundary and mixed boundary conditions. Math. Models Methods Appl. Sci. 7 (1997) 957-991.

[18] P. Fernandes and I. Perugia, Vector potential formulation for magnetostatic and modelling of permanent magnets. IMA J. Appl. Math. 66 (2001) 293-318.

[19] V. Girault and P. A. Raviart, Finite Element Methods for Navier-Stokes Equations. Theory and Algorithms. Springer-Verlag, Berlin (1986).

[20] R. Hiptmair and O. Sterz, Current and voltage excitations for the eddy current model. Int. J. Numer. Model. 18 (2005) 1-21.

[21] A. Kameari, Calculation of transient 3D eddy currents using edge elements. IEEE Trans. Magn. 26 (1990) $466-469$.

[22] A. Kameari, Three dimensional eddy current calculation using edge elements for magnetic vector potential. Applied Electromagnetic in Materials, Pergamon Press, Oxford (1988) 225-236.

[23] T. Kang, T. Chen, H. Zhang and K.I. Kim, Improved T $-\psi$ nodal finite element schemes for eddy current problems. Appl. Math. Comput. 218 (2011) 287-302.

[24] C. Ma, The finite element analysis of a decoupled $T-\Psi$ scheme for solving eddy-current problems. Appl. Math. Comput. 205 (2008) 352-361.

[25] G. Pichenot, F. Buvat, V. Maillot and H. Voillaume, Eddy current modelling for non destructive testing. Proc. of 16th World Conf. on NDT, Rapport DSR 31. Montreal, August 30 - September 3 (2004).

[26] B. Weiß and O. Bíró, On the convergence of transient eddy-current problems. IEEE Trans. Magn. 40 (2004) $957-960$.

[27] A. Ženíšek, Nonlinear Elliptic and Evolution Problems and their Finite Element Approximations. London, Academic Press (1990).

[28] W. Zheng, Z. Chen and L. Wang, An adaptive finite element method for the $H$ - $\psi$ formulation of time-dependent eddy current problems. Numer. Math. 103 (2006) 667-689. 